\title{
Multistability and complex basins in a nonlinear duopoly with price competition and relative profit delegation
}

\author{
Luciano Fanti* ${ }^{*}$ Luca Gori $^{\dagger} \bullet$ Cristiana Mammana ${ }^{\ddagger} \bullet$ Elisabetta Michetti ${ }^{\S}$
}

July 1, 2016

\begin{abstract}
In this paper we investigate the local and global dynamics of a nonlinear duopoly model with price-setting firms and managerial delegation contracts (relative profits). Our study aims at clarifying the effects of the interaction between the degree of product differentiation and the weight of manager's bonus on long-term outcomes in two different states: managers behave more aggressively with the rival (competition) under product complementarity and less aggressively with the rival (cooperation) under product substitutability. We combine analytical tools and numerical techniques to reach interesting results such as synchronisation and on-off intermittency of the state variables (in the cases of homogeneous attitude of managers) and the existence of chaotic attractors, complex basins of attraction and multistability (with heterogeneous attitudes of managers). We also give policy insights.
\end{abstract}

Keywords Bertrand duopoly; Local and global dynamics; Synchronisation and multistability; Relative profit delegation

JEL Codes C61; C62; D43; L13

AMS Codes $\quad 34 \mathrm{~A} 34 ; 70 \mathrm{~K} 50 ; 65 \mathrm{P} 20$

${ }^{*}$ L. Fanti, Department of Economics and Management, University of Pisa, Via Cosimo Ridolfi, 10, I-56124 Pisa (PI), Italy, e-mail: luciano.fanti@unipi.it, tel.: +390502216 369, fax: +390502210603.

${ }^{\dagger}$ L. Gori, Department of Political Science, University of Genoa, Piazzale E. Brignole, 3a, I-16125 Genoa (GE), Italy, e-mail: luca.gori@unige.it or dr.luca.gori@gmail.com, tel.: +39 01020995 03, fax: +39 0102095536.

${ }^{\ddagger}$ C. Mammana, Department of Economics and Law, University of Macerata, Via Crescimbeni, 20, I-62100 Macerata (MC), Italy, e-mail: cristiana.mammana@unimc.it.

${ }^{\S}$ E. Michetti (corresponding author), Department of Economics and Law, University of Macerata, Via Crescimbeni, 20, I-62100 Macerata (MC), Italy, e-mail: elisabetta.michetti@unimc.it. 
TITLE:

Multistability and complex basins in a nonlinear duopoly with price competition and relative profit delegation

\section{ABSTRACT:}

In this paper we investigate the local and global dynamics of a nonlinear duopoly model with price-setting firms and managerial delegation contracts (relative profits). Our study aims at clarifying the effects of the interaction between the degree of product differentiation and the weight of manager's bonus on long-term outcomes in two different states: managers behave more aggressively with the rival (competition) under product complementarity and less aggressively with the rival (cooperation) under product substitutability. We combine analytical tools and numerical techniques to reach interesting results such as synchronisation and on-off intermittency of the state variables (in the cases of homogeneous attitude of managers) and the existence of chaotic attractors, complex basins of attraction and multistability (with heterogeneous attitudes of managers). We also give policy insights.

The study of local and global properties in nonlinear dynamic oligopolies represents a relevant issue amongst the several recent advances of the theoretical economic literature. Economists often reject anything that departs from the hypothesis of rational expectations, essentially because of the linear models in which such an assumption is adopted. Anyway, the use of adaptive expectations or behavioural rules allow agents to go beyond the restriction implied by the rational expectations paradigm (i.e., a decisionmaker uses efficiently the set of available information) and it is useful to describe the nonlinear course of some economic variables (output, price and so on) observed in actual markets in both microeconomic and macroeconomic contexts. Since Bischi et al. (1998), the analysis of repeated nonlinear duopolies with profit-maximising firms or managerial firms that behave adptively has seen a burgeoning interest in the cases of both Cournot and Bertrand rivalries. This article contributes to this literature by considering a price competition game with managerial firms and relative profit contracts.

Keywords Bertrand duopoly; Local and global dynamics; Synchronisation and multistability; Relative profit delegation

JEL Codes C61; C62; D43; L13

AMS Codes $\quad 34 \mathrm{~A} 34 ; 70 \mathrm{~K} 50 ; 65 \mathrm{P} 20$

\section{INTRODUCTION}

Since Fama \& Jensen (1983), inquiring into the causes and consequences of the separation between ownership and management is the object of a growing body of studies. When governance of firms is different than perfect competition, the firm's objective is not just pure profit maximisation, so that owners may hire managers and motivate them by using (different kind of) incentives with the aim of achieving a competitive advantage in the market. The industrial organisation literature has pointed out the importance of the strategic use of managerial delegation contracts at least starting from Fershtman (1985), Vickers (1985), Fershtman \& Judd (1987) and Sklivas (1987). The structure of these models is represented by a two-stage game. In the first stage, owners choose a specific kind 
of incentive for managers (i.e., they select their own manager). In the second one, managers play a standard oligopoly game in the product market.

Stressing differences between ownership and control has then become a much debated issue, especially when developing delegation schemes in firms that operate under quantity competition or price competition, in both cases of homogeneous products and horizontal differentiation. The most important kinds of incentives for managers are based on a weighted average of profits and output (Vickers, 1985), profits and revenues (Fershtman 1985; Sklivas, 1987), profits and market shares (Jansen et al., 2007, 2009; Kopel \&Lambertini, 2013) and relative performance evaluation (SalasFumas, 1992; Miller \& Pazgal, 2001, 2002). The key result of this literature is that these incentives can strategically be used as devices to direct managers to be more aggressive in the market with the aim of increasing profits to a level higher than when firms behave just like quantity setters or price setters under standard profit maximisation. There exists a wide spectrum of outcomes that this literature has established with respect to which the society as a whole may be better off if firms hire managers instead of being profit maximisers (see, e.g., Fershtman \& Judd, 1987; Miller \& Pazgal, 2002).

In the particular case of relative profit delegation, which is the most controversial one but plays a preeminent role - together with the sales delegation - in the theoretical industrial economics literature, there is some empirical support that shows that top managers' compensation is actually based on this kind of contract (Gibbons \& Murphy, 1990; Barro \& Barro, 1990; Janakiraman et al., 1992; Aggarwal \&Samwick, 1999). With this regard, in a theoretical contribution Miller \& Pazgal (2002) have found that under price competition on product market and horizontal differentiation, there exists a relationship between the degree of product differentiation and the optimal value of the weight attached to manager's objective. In particular, contrary to Cournot competition, managers behave more (resp. less) aggressively when products are complements (resp. substitutes) under relative profits delegation and a competitive labour market. More recently, Meccheri \& Fanti (2014) have introduced unions in games where managers are compensated with incentives based on sales delegation and relative profit delegation, to study how this kind of contracts interact with a labour market where unions have the power to set the wage. In the case of relative profits, product differentiation and Cournot competition in the product market, they find that firms obtain larger (resp. smaller) profitability than in the standard case of profit maximisation when products are complements (resp. substitutes). In contrast, in the case of relative profits, product differentiation and Bertrand competition in the product market, profitability of firms is larger (resp. smaller) under profit maximisation than with relative profit delegation when products are complements (resp. substitutes). They also rank outcomes with regard to output, employment and social welfare by comparing sales delegation, relative profit delegation and profit maximisation.

Contextually to the development of the studies mentioned above, there exists a literature led by Puu (1991), Kopel (1996) and Bischi et al. (1998) that has deepened how oligopoly (profit maximising) firms behave in the long term in repeated discrete time models. ${ }^{1}$ Since their works, nonlinear oligopoly dynamics has become a much debated topic in either cases of complete information and static expectations and incomplete information with firms using specific behavioural rules to adapt production between two subsequent periods. The most important results of this literature are represented by the very complicated dynamics (endogenous fluctuations and chaos) that can be observed in markets where only two firms operate in the absence of exogenous stochastic shocks.

\footnotetext{
${ }^{1}$ See also Matsumoto \& Szidarovszky $(2012$, 2014) and Gori et al. (2015a) for the study of dynamic continuous time models with discrete delays.
} 
Given the importance of managerial firms in actual markets, the present article contributes to the nonlinear oligopoly literature and aims at studying how relative profit contracts affects long-term market dynamics in a model where players have incomplete information. Specifically, it concentrates on local and global dynamics in a repeated discrete time duopoly with managerial firms, price competition and horizontal differentiation by referring to the framework developed by Bischi et al. (1998), where players have limited information and use a behavioural rule to choose the strategic variable in the product market from one period to the subsequent one. It extends the work of Fanti et al. (2012) that has studied the effects of relative profit delegation contracts in a repeated duopoly with quantity-setting firms and homogeneous products. Indeed, differently from models with full rationality (i.e., agents are able to use efficiently information), where instability of equilibria is essentially related to the existence of exogenous stochastic shocks, building on models that formally take into account incomplete information of agents allows introducing some behavioural rules to overcome concerns related to high costs of obtaining and using information efficiently (Bischi et al., 1998, 2007). In this context, instability and fluctuations are endogenous to the model in the cases of both homogeneous and heterogeneous agents.

The present study develops a mathematical analysis applied to a discrete time dynamic model by using a theoretical framework commonly used in static games in the industrial organisation literature (Miller \& Pazgal, 2001, 2002). It also presents several numerical experiments to clarify the local and global properties of the dynamic system and gives some policy insights. The marginal bonus of each manager in our model drives the setting of prices from one period to the subsequent one. Results are given in two distinct cases, showing that the relationship between the degree of product differentiation and the weight of profits in the manager objective matters. In the first case the manager behaves more aggressively with the rival (competition) under product complementarity. In the second case the manager behaves less aggressively with the rival (cooperation) under product substitutability. In the former case (i.e., price competition with complementary goods) prices are strategic substitutes, while in the latter case (i.e., price competition with substitute goods) prices are strategic complements. When prices are strategic substitutes (resp. complements), competition (resp. cooperation) between managers tend to reduce (resp. increase) the marginal bonus of each manager. Since the mechanism that drives the setting of prices of managers is essentially related to each manager's marginal bonus, the degree of product differentiation and the attitudes of managers as well determine the long-term performance of the economy in a strategic context. In particular, the more managers' attitudes differ and/or the more products are complements or substitutes, the more likely endogenous fluctuations and complex basins of attraction may exist. This last phenomenon is of particular importance for the strategic behaviour of managers as it makes unpredictability a plausible scenario. If managers behave with an equal degree of cooperation or competition, we find that they may coordinate themselves (synchronisation) also when prices are strategic substitutes.

The present article represents a prosecution in a research agenda on nonlinear duopolies with price competition, horizontal product differentiation and managerial firms. In particular, it extends some results obtained in Fanti et. al $(2014,2015)$ by considering the case in which managers are paid according to relative profit delegation contracts. The existence of a delegation variable in a context with limited information is responsible for rich dynamic phenomena, as complex dynamics, multistability and complex basins, emerging when products are complements or substitutes. However, differently form the case of market share delegation contracts, when synchronised trajectories are owned, their qualitative behaviour is not affected by the marginal bonus.

The rest of the article proceeds as follows. Section 2 sets up the duopoly model with price 
competition, horizontal differentiation and managerial firms that behave according to relative profit contracts. Section 3 studies local and global dynamics in the general case of heterogeneous managers' types, showing some results related to endogenous fluctuations, coexistence of attractors and the complex structure of both attractors and basins of attraction. Section 4 concentrates on the specific case of homogeneous managers and stresses phenomena of synchronisation and intermittency. Section 5 outlines the conclusions and gives policy insights.

\section{THE MODEL}

The model describes the behaviour of consumers and producers (see Fanti et al., 2013, 2014 for details) in a duopoly with managerial firms that operate under relative profit contracts (Miller \& Pazgal, 2002; Meccheri \& Fanti, 2014), price competition and horizontal differentiation (Singh \& Vives, 1984). The modelling approach adopted here is basically the managerial delegation framework developed by van Witteloostuijn et al. (2007) and Jansen et al. (2009). Let $p_{i} \geq 0$ and $q_{i} \geq 0$ be firm $i$ 's price (the marginal willingness to pay of consumers) and quantity. Differently from profit maximising firms, under relative performance as a strategic commitment mechanism each firm hires a manager who receives a fixed salary (which is set to zero without loss of generality) plus a bonus offered in a publicly available contract and based on relative profit delegation. This is a contract that weights firm $i$ 's profit with the rival's one (firm $j$ ), $i, j=1,2, i \neq j$. As is common in this literature, we assume the nature of the remuneration as being exogenous to the model. This because it may be related to norms and rules typical of each single country (Jansen et al., 2009). Formally, when profits are positive the objective function of manager $i$ can be written as follows:

$$
W_{i}=\Pi_{i}-b_{i} \Pi_{j}, \quad i, j=1,2, \quad i \neq j,
$$

where $-1<b_{i}<1$ denotes manager $i$ 's attitude ("type"), $\Pi_{i}=\left(p_{i}-w\right) q_{i}$ are profits of firm $i$ and $0 \leq w<1$ is the constant average and marginal cost. This assumption implies that firm $i$ employs only labor in production and technology faces constant returns to scale, that is $q_{i}=L_{i}$, where $L_{i}$ represents the number of workers of firm $i$. When $b_{i}>0$ (resp. $b_{i}<0$ ), manager $i$ is more concerned in competition (resp. cooperation) with his rival. In the (static) related literature (Miller \& Pazgal, 2002; Meccheri \& Fanti, 2014), there exist at least two stages of the game to endogenously determine the optimal value of $b_{i}$ and the optimal value of the strategic variable (depending on whether firms are price setters or quantity setters). Specifically, in the first stage of the game each owner chooses the weight $b_{i}$ as part of the incentive contract of the manager. Then, in the second stage of the game each manager decides the output. In the particular case of price competition and relative profit delegation, there exists a specific relationship between the optimal value of weight $b_{i}$ and the degree of differentiation of products $d$, as is pointed out by Meccheri \& Fanti (2014, p. 60): "managers become more (less) aggressive when products are complements (substitutes)". This implies that if $d>0$ (resp. $d<0)$ then $b_{i}<0$ (resp. $b_{i}>0$ ) at the optimum. If products are independent (that is, $d=0)$ we get $b_{i}=0$.

Given the relevance of managerial firms and product differentiation in actual market, the main aim of the present article is to study the local and global dynamic properties of the model when the degree of differentiation of products $d$ or the delegation variable $b_{i}$ varies. To this purpose (and differently from the static literature on this issue), we consider $b_{i}$ as an exogenous parameter. However, by following Miller \& Pazgal (2002) and Meccheri \& Fanti (2014), in what follows we assume (in line with the results of the static literature on this issue) that managers behave more 
(resp. less) aggressively under product complementarity (resp. substitutability). Then, the following remark holds.

Remark 1. If $d>0$ (resp. $d<0)$ then $b_{i}<0$ (resp. $\left.b_{i}>0\right)$.

Remark 1 implies that - given price competition - when products are substitutes (resp. complements) managers should cooperate (resp. compete) between each other, that is in the former cases prices are strategic complements, while in the latter case prices are strategic substitutes. Some clarifications on the reasons why the delegation variable $b_{i}$ is a constant in this kind of dynamic models with limited knowledge are now in order. In static games of the managerial delegation literature, where players have full information and the link owner-manager interacts to market competition in a strategic context, $b_{i}$ is a control variable chosen by owner $i$ in the first stage of the game (also known as the contract stage). Knowing all the weights chosen in the contract stage by the owners, managers choose the quantity or the price (depending on the nature of the game) in order to maximise their own utilities in the second stage of the game (also known as the market stage). In these games, owners and managers choose simultaneously and independently and the game is definitely is solved by using the backward logic. In this work, however, we consider $b_{i}$ as an exogenous parameter. In fact, in our dynamic model players do not have perfect knowledge. Then, managers do not compute their own best replies in the market stage (as they do not know the owners' choices about the the delegation variable) and owners do not compute the optimal value of $b_{i}$. Therefore, given the assumption of limited information, it would be of importance for managers (as will be clear when we will introduce the behavioural adjustment mechanism in Eq. (5)) to know the direction of the change in output given $b_{i}$ and the other parameters of the model.

We now briefly sketch the behaviour of consumers. By assuming that consumers' preferences are expressed by the usual utility function (see Singh \& Vives, 1984; Häckner, 2000),

$$
U\left(q_{1}, q_{2}\right)=q_{1}+q_{2}-\frac{1}{2}\left(q_{1}^{2}+q_{2}^{2}+d q_{1} q_{2}\right),
$$

the indirect demand for product of firm $i$ is $p_{i}=1-q_{i}-d q_{j}(i, j=1,2, \quad i \neq j)$. Therefore, the direct demand is the following:

$$
q_{i}=\frac{1-p_{i}-d\left(1-p_{j}\right)}{1-d^{2}}, \quad i, j=1,2, \quad i \neq j
$$

where $-1<d<1$ is the degree of horizontal product differentiation. When $d>0$ (resp. $d<0$ ) products are substitutes (resp. complements). Definitely, parameter $d$ captures how consumers perceive products as being differentiated between each other. We consider $d$ as exogenously given parameter. However it is important to recall that $d$ may be affected by the level of advertising investment, as pointed out by Gori et al. (2015b).

By setting $w=0$ without loss of generality and knowing that price is the manager's control variable, the marginal bonus of $i$ th player is given by:

$$
\frac{\partial W_{i}}{\partial p_{i}}=\frac{1-2 p_{i}-d\left(1-p_{j}\right)-b_{i} d p_{j}}{1-d^{2}}
$$

We now assume a discrete time dynamic setting where time is indexed by $t \in \mathbb{Z}_{+}$. By assuming players with limited information as in Bischi et al. (1998), i.e. managers do not have enough 
information to know each other's type (because of the high costs associated with full information), the behavioural rule used for the setting of price from time $t$ to time $t+1$ is the following:

$$
p_{i}^{\prime}=p_{i}+\alpha p_{i} \frac{\partial W_{i}}{\partial p_{i}},
$$

where $/$ is the unit-time advancement operator and $\alpha>0$. The assumption of limited information and adaptive behavioural rules of agents is made up to overcome problem of choosing in contexts where costs of obtaining and using information efficiently are high (Léonard \& Nishimura, 1999). Although it has been intensely criticised, the assumption of adaptive expectations may well capture the essence of nonlinear economic models, as is well pointed out by Agliari et al. (2006, p. 527): "What the arguments against adaptive expectations overlooked was the fact that the linear models frequently used in economics are usually an approximation to some underlying nonlinear model. If parameter sets for which the steady state is locally unstable are admitted, then as the paths move sufficiently far from the steady state the linear approximation breaks down and one needs to consider the nonlinear nature of the economic mechanism in order to obtain a true picture of the dynamics. When one takes into account the fact that nonlinear dynamic systems can produce dynamic paths that are not so regular and predictable, one of the major arguments against adaptive expectations does not seem so strong". This may have policy consequences, especially in the study of global dynamics. Economic differences and similarities that come from the use of (5) in models with quantity-setting firm and price-setting firms have been discussed in Brianzoni et al. (2015). We refer to such a work for more details.

By using (3), (4) and (5) the discrete time two-dimensional system that characterises the dynamics of prices in this model is:

$$
\left\{\begin{array}{l}
p_{1}^{\prime}=p_{1}+\alpha p_{1}\left[\frac{1-2 p_{1}-d\left(1-p_{2}\right)-b_{1} d p_{2}}{1-d^{2}}\right] \\
p_{2}^{\prime}=p_{2}+\alpha p_{2}\left[\frac{1-2 p_{2}-d\left(1-p_{1}\right)-b_{2} d p_{1}}{1-d^{2}}\right] .
\end{array} .\right.
$$

\section{LOCAL AND GLOBAL DYNAMICS}

Define $x^{\prime}=p_{1}^{\prime}, x=p_{1}, y^{\prime}=p_{2}^{\prime}$, and $y=p_{2}$. The discrete time two-dimensional dynamic system that characterises the evolution of prices of each variety in the Bertrand duopoly with horizontal differentiation and relative profit delegation is given by:

$$
T:\left\{\begin{array}{l}
x^{\prime}=x F(x, y)=x\left[1+\alpha\left(\frac{(1-d)-2 x+d\left(1-b_{1}\right) y}{1-d^{2}}\right)\right] \\
y^{\prime}=y G(x, y)=y\left[1+\alpha\left(\frac{(1-d)-2 y+d\left(1-b_{2}\right) x}{1-d^{2}}\right)\right]
\end{array},\right.
$$

where $\alpha>0, d \in(-1,1), b_{i} \in(-1,1)$, and $b_{i} d \in(-1,0), i=1,2$. In what follows we describe both the qualitative and quantitative long-term dynamics produced by $(T, Q)$ where $Q \subset \mathbb{R}_{+}^{2}$ is the convex polygon with vertices $(0,0),(0,1-d),(1,1)$ and $(1-d, 0)$, since prices and quantities of products of both varieties must be non-negative.

\subsection{The structure of the feasible set}

As it has been stressed, we are interested in the dynamics produced by system (7) for any initial condition belonging to $Q$. Obviously, a trajectory is economically meaningful only whether, at any time $t$, the two prices $x$ and $y$ belong to $Q$. We pursue the following definition (see also Fanti et al. 2014 and 2015). 

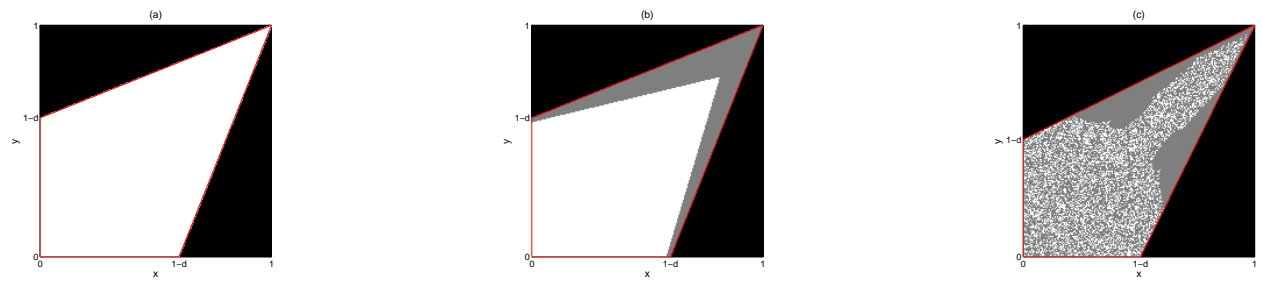

Figure 1: (a) The feasible set $D=Q$ is depicted in white for $\alpha=0.5, d=0.4, b_{1}=-0.5, b_{2}=-0.2$. (b) For $\alpha=1.5$ and the other parameters as in $(a)$, the feasible set $D \subset Q$ is depicted in white while the gray points are initial conditions producing unfeasible trajectories. (c) For $\alpha=1.5, b_{1}=-0.95$, $b_{2}=-0.9$ and $d=0.4933$ the feasible set has a complex structure.

Definition 1. Let $T^{t}(x(0), y(0)), t=0,1,2 \ldots$, denote the $t$-th iterate of system $T$ for a given initial condition $(x(0), y(0)) \in Q$. Then, the sequence $\{(x(t), y(t))\}_{t=0}^{\infty}$ is called trajectory. A trajectory is said to be feasible for system $T$ if $(x(t), y(t)) \in Q$ for all $t \in \mathbb{N}$, otherwise it is unfeasible. The set $D \subseteq Q$ whose points generate feasible trajectories is called feasible set and a point $(x(0), y(0)) \in D$ is a feasible point.

It can immediately be verified that the origin is a feasible point for all parameter values, while, a threshold value $\bar{\alpha}$ does exist such that if $\alpha>\bar{\alpha}$ then $(1,1)$ is not a feasible point. As a consequence, the feasible set is non-empty but unfeasible trajectories may be produced.

In Figure 1 the feasible set for different parameter constellations is depicted in white, while the grey points represent initial conditions that belong to $Q$ and produce unfeasible trajectories. Notice that in panel $(a)$ the whole set $Q$ is feasible, while in panels $(b)$ and $(c)$ set $D \subset Q$. Furthermore, in panels $(a)$ and $(b)$ the feasible set has a simple structure (connected set), while in panel $(c)$ it has a complex structure (infinitely many non-connected sets). As a consequence, in order to assess the feasibility of system $T$, two questions must be investigated: $(i)$ the existence of conditions such that all (resp. not all) points in $Q$ are feasible, and $(i i)$ how the structure of the feasible set changes from simple to complex as parameters vary. With regard to question $(i)$ the following results can be proved.

Proposition 1. Let $T$ be given by system (7).

(i) If $d \rightarrow 1^{-}$then $D \rightarrow\{(0,0)\}$.

(ii) If $d \rightarrow-1^{+}$then $D-\left\{(0,0),(0,1),(1,0),\left(\frac{2\left[2-\left(1-b_{1}\right)\right]}{4-\left(1-b_{1}\right)\left(1-b_{2}\right)}, \frac{2\left[2-\left(1-b_{2}\right)\right]}{4-\left(1-b_{1}\right)\left(1-b_{2}\right)}\right)\right\}$ is empty.

(iii) If $\alpha \rightarrow+\infty$ then there exists $x_{1} \in(0,1-d], y_{1} \in(0,1-d]$ and $\left(x^{*}, y^{*}\right) \in Q \cap \mathbb{R}_{+}^{2}$ such that $D-\left\{(0,0),\left(0, y_{1}\right),\left(x_{1}, 0\right),\left(x^{*}, y^{*}\right)\right\}$ is empty.

Proof. ( $i$ ) Firstly notice that $(0,0)$ is always feasible. Secondly observe that if $d \rightarrow 1^{-}$, then the convex polygon $Q$ tends to the segment $I=\{(x, x): x \in[0,1]\}$. Hence, to prove the statement, we consider an initial condition $(x(0), y(0))$ such that $x(0)=y(0)$ and $x(0) \in(0,1]$ and we show that it produces an unfeasible trajectory. By taking into account the first equation in system (7), if $d \rightarrow 1^{-}$we obtain

$$
\lim _{d \rightarrow 1^{-}} x(1)=-\infty
$$

being $-1-b_{1}<0$. This means that $(x(0), y(0))$ exits from $Q$ at the first iteration. 
(ii) If $d \rightarrow-1^{+}$then $Q \rightarrow I=\left\{(x, y) \in \mathbb{R}_{+}^{2}: x+y \leq 2, x \geq 0, y \geq 0\right\}$. Since $(0,0) \in D$, we consider initial conditions such that at least one component of $(x(0), y(0))$ is strictly positive. Consider firstly the case in which $y(0)=0$ and $x(0) \in(0,2]$. Then

$$
\lim _{d \rightarrow-1^{+}} x(1)=\infty \text { if } x(0) \neq 1 .
$$

A similar reasoning holds if $x(0)=0$ and $y(0) \in(0,2]$.

Secondly, let $x(0) \in(0,2]$ and $y(0) \in(0,2]$. Then

$$
\lim _{d \rightarrow-1^{+}} x(1)=\infty \text { if } 2(1-x(0))+\left(b_{1}-1\right) y(0) \neq 0
$$

and, similarly,

$$
\lim _{d \rightarrow-1^{+}} y(1)=\infty \text { if } 2(1-y(0))+\left(b_{2}-1\right) x(0) \neq 0 .
$$

Since $2(1-x(0))+\left(b_{1}-1\right) y(0)=2(1-y(0))+\left(b_{2}-1\right) x(0)=0$ iff $x=x^{*}=\frac{2\left[2-\left(1-b_{1}\right)\right]}{4-\left(1-b_{1}\right)\left(1-b_{2}\right)}$ and $y=y^{*}=\frac{2\left[2-\left(1-b_{2}\right)\right]}{4-\left(1-b_{1}\right)\left(1-b_{2}\right)}$, then the statement is proved.

(iii) Let $(x(0), y(0)) \in Q$. Then

$$
\lim _{\alpha \rightarrow+\infty} x(1)=\lim _{\alpha \rightarrow+\infty} x(0)\left[1+\alpha \gamma_{1}\left(d, b_{1}, x(0), y(0)\right)\right]=\infty
$$

as long as $x(0) \neq 0$ and $\gamma_{1}\left(d, b_{1}, x(0), y(0)\right) \neq 0$.

Similarly,

$$
\lim _{\alpha \rightarrow+\infty} y(1)=\lim _{\alpha \rightarrow+\infty} y(0)\left[1+\alpha \gamma_{2}\left(d, b_{2}, x(0), y(0)\right)\right]=\infty
$$

as long as $y(0) \neq 0$ and $\gamma_{2}\left(d, b_{2}, x(0), y(0)\right) \neq 0$.

For all parameter values, it can be easily verified that $\exists ! x_{1} \in(0,1-d]$ such that $\gamma_{1}\left(d, b_{1}, x_{1}, 0\right)=$ 0 and that $\exists ! y_{1} \in(0,1-d]$ such that $\gamma_{2}\left(d, b_{2}, 0, y_{1}\right)=0$; futhermore $\gamma_{1}\left(d, b_{1}, x^{*}, y^{*}\right)=$ $\gamma_{2}\left(d, b_{2}, x^{*}, y^{*}\right)=0$ in a unique point $\left(x^{*}, y^{*}\right) \in Q \cap \mathbb{R}_{+}^{2}$. The statement is then proved.

According to the previous Proposition, if products are too much complements or substitutes (i.e. $d$ is close to its extreme values) then almost all trajectories are unfeasible, thus confirming the results proved in Fanti et al. (2013) (without delegation) and Fanti et al. (2014, 2015) (with market share delegation). Similarly, $D$ is comprised only of a finite number of points also if the speed of adjustment of prices with respect to the marginal bonus is high. For these reasons, economically meaningful long-term dynamics can be produced only for intermediate values of $d$ and a sufficiently low value of $\alpha$.

From an economic point of view, it is important to stress that - under price competition - there exists a specific relationship between the extent of product differentiation and the weight attached to rival's profits in the manager's objective function. In fact, owners want that the manager competes (resp. cooperates) with the rival under product complementarity (resp. substitutability), because prices are strategic substitutes (resp. complements) in that case. In order to have feasible trajectories 1) products should not be too much complements or substitutes, and 2) the weight attached rival's profits in the manager's bonus should not be fixed at too high a level. Therefore, advertising policies aiming at increasing the degree of competition or cooperation between two firms by favoring production of goods that are complements enough (resp. homogeneous goods) may cause the exit 
from the market of at least one firm. The same result applies as long as firms' owners try to motivate their managers to behave either aggressively or cooperatively in the market for achieving an adequate competitive advantage with respect to the rival. In both cases, in fact, marginal profits increase or reduce too much and firm $i$ would overreact by fixing a price for the subsequent period that causes the exit from the market.

With regard to the second question, i.e. how the structure of $D$ changes as some parameters are moved, we underline that the study of the structure of set $D$ is of importance both from an economic perspective and a mathematical perspective since the long-term evolution becomes path-dependent, and a thorough knowledge of the properties of $D$ becomes crucial in order to predict the feasibility of the economic system. Of course, this may have relevant policy consequences.

The critical curves technique, proposed for the first time by Mira et al. (1996) and Abraham et al. (1997), represents a numerical procedure that can be used to describe some global properties of a differentiable map on the plane. We will use this method to describe the boundary of the feasible set and its modifications as some parameters are changed (see also Bischi et al., 2000 and Brianzoni et al., 2009).

We recall that the critical curve of rank-1, denoted by $L C$, is the set of points having two, or more, coincident rank-1 preimages, located on a set denoted by $L C_{-1}$, called curve of merging preimages. Arcs of LC separate set $Q$ into regions characterised by a different number of preimages and the locus $L C_{-1}$ is given by the set of points such that $|J(x, y)|=0$, where $J(x, y)$ is the Jacobian matrix of map $T$ :

$$
\begin{gathered}
J(x, y)= \\
\left(\begin{array}{ll}
1+\alpha\left(\frac{1-d-4 x+d\left(1-b_{1}\right) y}{1-d^{2}}\right) & \alpha \frac{d\left(1-b_{1}\right)}{1-d^{2}} x \\
\alpha \frac{d\left(1-b_{2}\right)}{1-d^{2}} y & 1+\alpha\left(\frac{1-d-4 y+d\left(1-b_{2}\right) x}{1-d^{2}}\right)
\end{array}\right) .
\end{gathered}
$$

The set of points such that $|J(x, y)|=0$ is depicted in Figure $2(a)$, while curves $L C=T\left(L C_{-1}\right)$ separate polygon $Q$ into regions having a different number of preimages (see Figure $2(b)$ ).

Consider the following parameter values $\alpha=0.5, b_{1}=0.2$ and $b_{2}=0.4$ then the complexity of the feasible set $D$ increases when parameter $d$ is decreased (i.e. complementarity between products increases). As is shown in Figure $2(a)$, if $d=-0.8$ set $D$ has a simple structure. However, it can easily be observed that $L C^{b}$ curve moves upwards as parameter $d$ decreases so that, given the other parameter values, a threshold value $\bar{d} \simeq-0.821$ does exist such that a contact between a critical curve and the boundary of the feasible set occurs (see Figure $2(c)$ ), and new components of the unfeasible set suddenly appear after the contact (see Figure $2(d)$ ).

It can be also observed that, after the contact bifurcation, the complexity of the structure of the feasible set increases if $d$ further decreases and the gray area increases as well, as it is shown in Figure $2(e)$, until a final bifurcation occurs at $d \simeq-0.82315$ after which almost all trajectories become unfeasible (see Figure $2(f)$ ), as proved in Proposition 1 (ii).

This result of course may have dramatic economic consequences. In fact, one can think about advertising policies aiming at affecting the degree of product differentiation through an increase in product complementarity. In a Bertrand duopoly with managerial firms and relative profit contracts, this causes an increase in the degree of competition between managers when prices are strategic substitutes. These polices, however, may have the unpleasant effect of moving the economy towards initial conditions that generate unfeasible trajectories.

The numerical simulations presented in Figure 2 describe the bifurcations occurring in the structure of set $D$ if $d$ is negative (i.e., products are complements). It can be also observed that a similar 

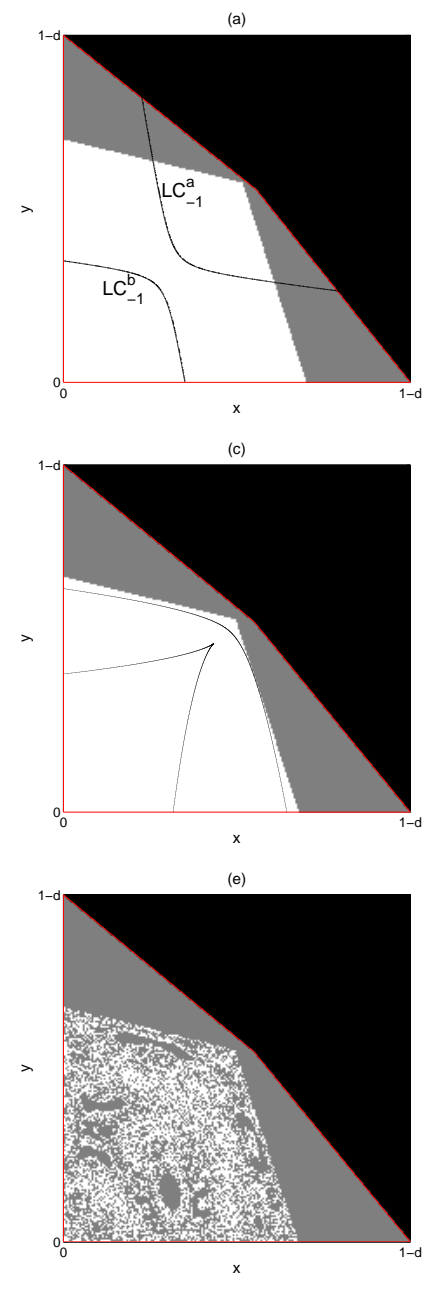
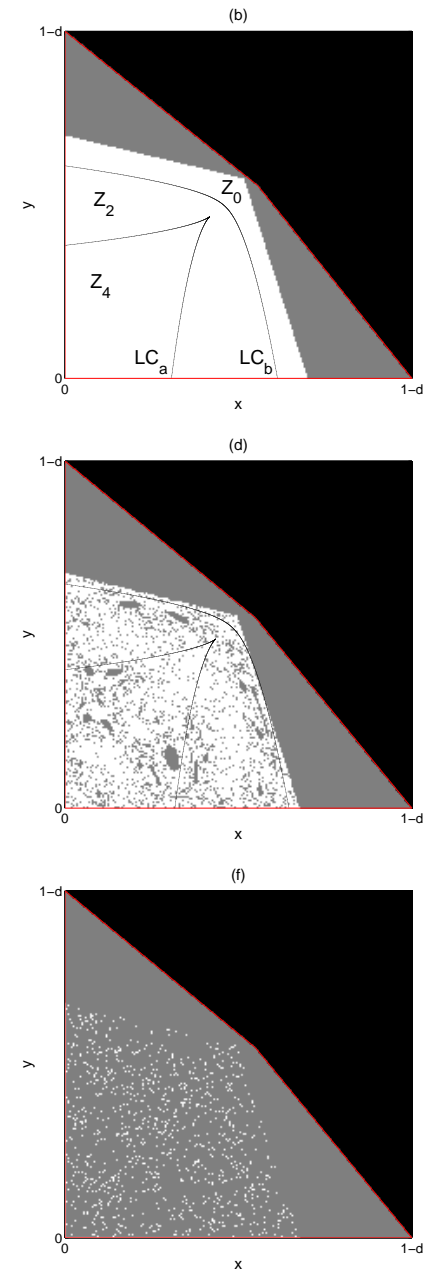

Figure 2: Parameter values: $\alpha=0.5, b_{1}=0.2$ and $b_{2}=0.4$. (a) Critical curves of rank-0, $L C_{-1}$, for system $T$ and $d=-0.8$. (b) Critical curves of rank-1, $L C=T\left(L C_{-1}\right)$, for the same parameter values as in panel $(a)$. (c) Immediately before the contact bifurcation $(d \simeq-0.821)$, the tangency between the critical curve and the boundary of the feasible set is shown. (d) Set $D$ immediately after the contact bifurcation $(d=-0.822)$ : gray holes are depicted. $(e) D$ has a complex structure $(d=-0.8228) .(f)$ Immediately before the final bifurcation $(d=-0.82315)$ almost all trajectories are unfeasible. 
behavior holds also if $d$ is positive (i.e. under substitutability between products that implies that prices are strategic complements). More precisely, it is possible to observe that the structure of the feasible set increases in complexity when moving away from the hypothesis of independence between products.

Given the analytical and numerical results reached in this subsection, in what follows we will focus on the study of the dynamics produced by $T$ by assuming that $\alpha$ is fixed at low level and $d$ ranges between intermediate values.

\subsection{Fixed points and invariant sets: local stability and multistability}

In order to describe the long-term dynamics produced by system $T$, it is important to determine its fixed points and other invariant sets.

Definition 2. Let $T$ be given by system (7). A point $(\bar{x}, \bar{y}) \in Q$ is a fixed point of $T$ if $T(\bar{x}, \bar{y})=$ $(\bar{x}, \bar{y})$; a set $S \subset Q$ is said to be invariant if $T(S)=S$.

Taking into account the previous definitions, the following results can be easily proved.

Proposition 2. Let $T$ be given by (7). Then:

(i) $T$ admits four fixed points for all parameter values, they are given by $E_{0}=(0,0), E_{1}=$ $\left(\frac{1-d}{2}, 0\right), E_{2}=\left(0, \frac{1-d}{2}\right)$ and $E^{*}=\left(x^{*}, y^{*}\right)=\left(\frac{(1-d)\left[2+d\left(1-b_{1}\right)\right]}{4-d^{2}\left(1-b_{1}\right)\left(1-b_{2}\right)}, \frac{(1-d)\left[2+d\left(1-b_{2}\right)\right]}{4-d^{2}\left(1-b_{1}\right)\left(1-b_{2}\right)}\right) ;$

(ii) $T$ admits the following invariant sets for all parameter values, $I_{x}=\{(x, y) \in D: x \in$ $[0,1-d], y=0\}$ and $I_{y}=\{(x, y) \in D: y \in[0,1-d], x=0\}$; the dynamics of $T$ on such segments are governed by the one-dimensional maps $\phi_{x}(x)=x\left[1+\alpha \frac{1-d-2 x}{1-d^{2}}\right]$ and $\phi_{y}(y)=$ $y\left[1+\alpha \frac{1-d-2 y}{1-d^{2}}\right]$

(iii) if $b_{1}=b_{2}=b$, then also the set $\Delta=\{(x, y) \in D: x \in[0,1], y=x\}$ is invariant for $T$ and the dynamics of $T$ on such a segment are governed by the one-dimensional map $\phi(x)=$ $x\left[1+\alpha \frac{1-d+[d(1-b)-2] x}{1-d^{2}}\right]$.

Notice that fixed points $E_{0}, E_{1}$ and $E_{2}$ are located on the invariant coordinate axes and that $E_{1}$ and $E_{2}$ are in symmetrical positions with respect to the main diagonal. Furthermore, $E^{*}$ is the unique interior Nash equilibrium of the game and it is feasible for all parameter values. We now want to consider how changes in the parameters of interest affect the Nash equilibrium price levels. First, by comparing $x^{*}$ to $y^{*}$, it is possible to observe that the following Proposition holds.

Proposition 3. Let products be substitutes (resp. complements), that is $d>0$ (resp. $d<0$ ). Then $x^{*} \geq y^{*}$ iff $b_{1} \leq b_{2}$ (resp. $\left.b_{1} \geq b_{2}\right)$.

Similar to the case of market share contracts studied in Fanti et al. (2014), when managers are of different kinds the Nash equilibrium values of prices are different. This proposition implies that the lower price is associated with the firm where the manager behaves more cooperatively with the rival (high degree of cooperation) when products are substitutes, because benefits of higher profits can be shared more easily between the two managers in this case. In contrast, when products are complements the lower price is associated with the firm where the manager behaves less aggressively with the rival (low degree of competition). In fact, in this case a firm wants to set a lower price to let also the product of the rival be more competitive and get higher profits because of complementarity. 
In both cases of substitutability and complementarity, therefore, there is a sort of cooperation between managers that choose the price in managerial firms according relative profits delegation contracts. When products are substitutes, owners drive managers towards a cooperative behaviour with the rival because profits are higher in that case (we recall that prices are strategic complements in this case), while when products are complements owners prefer that managers compete with the rival to share the benefit of complementarity (we recall that prices are strategic substitutes in this case).

A second question that arises is related to changes of the equilibrium price levels as the managers' attitude $\left(b_{i}\right)$ or the degree of horizontal differentiation between products $(d)$ varies. The following Proposition can then be proved.

Proposition 4. Let $T$ be given by system (7).

(i) If $d>0$ then $x^{*}$ and $y^{*}$ decrease as $d$ increases.

(ii) If $d \in I_{-}(0)$, where $I_{-}(0)$ denotes the left neighborhood of 0 , then $x^{*}$ and $y^{*}$ decrease as $d$ increases.

(iii) If $d>0$ (resp. $d<0)$ then $x^{*}$ decreases (resp. increases) as $b_{1}$ increases and $y^{*}$ decreases (resp. increases) as $b_{2}$ increases.

(iv) For all $d \in(-1,1), x^{*}$ is decreasing with respect to $b_{2}$ and $y^{*}$ is decreasing with respect to $b_{1}$.

Proof. $\quad(i)$ We rewrite $x^{*}$ as follows:

$$
x^{*}=\frac{-\left(1-b_{1}\right) d^{2}-\left(1+b_{1}\right) d+2}{4-d^{2}\left(1-b_{1}\right)\left(1-b_{2}\right)}
$$

then

$$
\frac{\partial x^{*}}{\partial d}=\frac{-\left(1-b_{1}^{2}\right)\left(1-b_{2}\right) d^{2}-4\left(1-b_{1}\right)\left(1+b_{2}\right) d-4\left(1+b_{1}\right)}{M}
$$

where $M>0$. Since $\left(1-b_{i}\right) \in(0,2)$ and $\left(1+b_{i}\right) \in(0,2), \forall b_{i} \in(-1,1), i=1,2$, then if $d>0$, $\frac{\partial x^{*}}{\partial d}<0$. Similarly it can be proved that if $d>0$, also $\frac{\partial y^{*}}{\partial d}<0$.

(ii) Since $\frac{\partial x^{*}}{\partial d}$ given by (9) is continuos w.r.t. $d$ and $\left.\frac{\partial x^{*}}{\partial d}\right|_{d=0}=\frac{-4\left(1+b_{1}\right)}{M}<0$, then $\exists I_{-}(0)$ such that $\frac{\partial x^{*}}{\partial d}<0, \forall d \in I_{-}(0)$. Trivially the same reasoning holds also for $y^{*}$.

(iii) Notice that

$$
\frac{\partial x^{*}}{\partial b_{1}}=\frac{-2 d(1-d)\left(2+d\left(1-b_{2}\right)\right)}{M}
$$

where $M>0$. It can be easily observed that if $d>0$ then $\frac{\partial x^{*}}{\partial b_{1}}<0$, that is $x^{*}$ is decreasing w.r.t. $b_{1}$, while, if $d<0$ then $\frac{\partial x^{*}}{\partial b_{1}}>0$ hence $x^{*}$ increases as $b_{1}$ increases. The same relations hold for $y^{*}$ while considering parameter $b_{2}$.

(iv) Simple computations show that $\frac{\partial x^{*}}{\partial b_{2}}<0$ and $\frac{\partial y^{*}}{\partial b_{1}}<0$, for all $d \in(-1,1)$.

From parts $(i)$ and $(i i)$ of the Proposition 4 it can be observed that, if products are substitutes or they are not too much complements (that is $d$ is negative but not too small), the equilibrium price levels decrease as $d$ increases given the other parameters of the system. This is quite intuitive to be explained. In fact, when products are substitutes managers cooperate between each other 
to share the benefits of higher profits (i.e., $b_{1}$ and $b_{2}$ are negative). Therefore, an increase in the degree of substitutability causes a reduction in prices because competition between products of firm 1 and firm 2 increases. The weight of this price war cannot be compensated by the cooperative behaviour of managers. Since the effect of $d$ on prices is nonlinear, the same line of reasoning applies if products are no more substitutes but not too much complements. In this case, in fact, managers compete between each other (i.e., $b_{1}$ and $b_{2}$ are positive). However, a reduction in the degree of complementarity ( $d$ increases) tends to reduce prices if products are not too much complements because the weight of competition between managers more than compensates the effect of a change of $d$ on prices in this case.

However, if $d$ is negative and small enough (products are sufficiently complements) from Proposition 4 we have that:

$$
\frac{\partial x^{*}}{\partial d} \rightarrow \frac{-\left(1-b_{1}\right)\left(1+b_{1}\right)\left(1-b_{2}\right)+4\left(1-b_{1}\right)\left(1+b_{2}\right)-4\left(1+b_{1}\right)}{M},
$$

if $d \rightarrow-1^{+}$. Hence, depending on the values of the two coefficients $b_{1}$ and $b_{2}$ the sign of $\frac{\partial x^{*}}{\partial d}$ can be positive or negative if $d$ is close to -1 . For instance, it can be observed that if $b_{2}$ is sufficiently high and $b_{1}$ is small enough, then $x^{*}$ may increase with $d$ if the degree of complementarity between products is high ( $d$ low enough). A similar line of reasoning holds for $y^{*}$ if $b_{2}$ is small and $b_{1}$ is high. As expected, therefore, the role of $d$ and $b_{i}$ are strictly related. In other words, if both managers compete (complementarity between products) but the one who is employed in the rival firm competes with a higher degree than its own manager does, then the price of its own product may actually increase when the degree of complementarity reduces if it is initially high. This because the weight of competition of the rival offsets the weight of the reduction in the degree of complementarity at the Nash equilibrium, as the degree of cooperation of the manager is relatively small. This actually causes the unpleasant effect of increasing the price of its own product, with potential negative consequences also for the rival. We recall that a reduction in the price of one good causes an increase in the quantity produced and sold by that firm, but reduces (resp. increases) the one of rival if products are substitutes (resp. complements).

By contrast, point (iii) of Proposition 4 allows us to study the role of managers' attitude on equilibrium prices. In particular, in order to reduce the price of product $1\left(x^{*}\right)$ it is necessary to reduce (resp. increase) $b_{1}$ if products are complements (resp. substitutes). This is quite intuitive. Indeed, in order to increase their own utility managers should behave less aggressively (reduce the degree of competition) in the case of complementarity. Alternatively, they should behave cooperatively (increase the degree of cooperation) in the case of substitutability. If one think about advertising policies (that directly affect the degree of product substitutability), it is immediate to understand the implications for managers and decision makers given the importance of changes that these variable may cause on long-term outcomes. Finally, by considering the role of one manager type on the price of product of the other firm, it can be observed that each equilibrium price is decreasing with respect to the other manager type (point (iv) of Proposition 4). This holds exactly for the opposite reasons as those detailed for the result summarised in point (iii) above.

The local stability analysis of the four fixed points of $T$ can be carried out by considering the Jacobian matrix associated with system $T$ given by (8). By considering $J(x, y)$ evaluated at a fixed point, and taking into account the fact that the eigenvalues of a diagonal or a triangular matrix are given by the elements of the main diagonal, then it can easily be observed that $E_{0}$ is an unstable node, while $E_{1}$ and $E_{2}$ can be both unstable nodes or saddle points (in this case the invariant axes are the stable manifolds of the saddle points). 
On the other hand, for what it concerns the dynamics embedded into the two invariant sets $I_{x}$ and $I_{y}$, those are subsets of the two invariant semi-axes, by following a line of reasoning similar to the one proposed in Fanti et al. (2013), it can be observed that maps $\phi_{x}$ and $\phi_{y}$ are topologically conjugated to the standard logistic map $\omega^{\prime}=\lambda \omega(1-\omega)$ through a linear transformation. Thus, the dynamics generated by map $T$ on the two invariant sets $I_{x}$ and $I_{y}$ are completely known, as these can be obtained from those of the logistic map. In particular, as long as an attractor $A$ on these restrictions exists, then it increases in complexity when parameter $d$ tends to \pm 1 . However, by considering the eigenvalues of $J(x, 0)$ and $J(0, y)$, it can easily be observed that sets $I_{x}$ and $I_{y}$ are repellor so that they cannot attract any (economic meaningful) initial condition $(x(0), y(0))$ having both positive components. For this reason, in the rest of the article we focus on the dynamics produced by system $T$ on $\mathbb{R}_{+}^{2} \cap D$.

Given the analytical form of the Jacobian matrix and that of the interior fixed point, the local stability analysis of $E^{*}$ is quite difficult to be studied analytically. Nevertheless, some results can be obtained by focusing on some limiting cases, while more general considerations can emerge by using numerical techniques.

About the local stability of Nash equilibrium $E^{*}$ in the limiting case in which $d$ is close to zero, the following Proposition can be proved.

Proposition 5. Let $T$ be given by map (7). Then $\forall \alpha \in(0,2), \exists I(0)$ such that $E^{*}$ is locally stable $\forall d \in I(0)$.

Proof. Consider the Jacobian matrix $J(x, y)$ given by (8). Then if $d \rightarrow 0$, both $x^{*} \rightarrow 1 / 2$ and $y^{*} \rightarrow 1 / 2$, furthermore

$$
J\left(x^{*}, y^{*}\right) \rightarrow\left(\begin{array}{ll}
1-\alpha & 0 \\
0 & 1-\alpha
\end{array}\right)
$$

which is a diagonal matrix with eigenvalues $\lambda_{1}=\lambda_{2}=\lambda=1-\alpha$. Observe that if $\alpha \in(0,2)$ then $\lambda \in(-1,1)$ and consequently $E^{*}$ is locally stable as long as $d$ belongs to a suitable neighborhood of the origin.

Proposition 5 is related to the local stability of the Nash equilibrium if the degree of horizontal differentiation between products is low enough, that is $d$ is close to zero. In this case, for all values of manager's types $b_{1}$ and $b_{2}, E^{*}$ is locally stable as long as $\alpha$ is not too high. In addition, several numerical experiments show that in this case $E^{*}$ is also globally stable, in the sense that it attracts every initial conditions taken on the interior of the feasible set $D$, representing economic meaningful initial conditions.

Furthermore, it can be verified that if $\alpha \rightarrow 0$ then the $d$-interval corresponding to the local stability of $E^{*}$ tends to $(-1,1)$, while such an interval gets smaller as $\alpha$ increases. This can be ascertained by comparing the bifurcation diagrams in Figure $3(a)$ obtained for two different values of $\alpha$. This result is in line with the related literature (Bischi et al., 1998, 1999). Parameter $\alpha$, in fact, weights the speed of adjustment of firm $i$ 's price from time $t$ to time $t+1$ given a marginal change in the manager's bonus. Ceteris paribus, a higher value of $\alpha$ induce firms to overreact (increase or reduce) given the value of the marginal bonus (positive or negative).

Another interesting question that can be considered is related to the case in which $\alpha \notin(0,2)$ and $d$ is close to zero. A preliminary consideration is that if $d=0$ and $\alpha$ crosses 2 then both $\lambda_{1}$ and $\lambda_{2}$ simultaneously cross -1 and consequently a flip bifurcation occurs causing two locally stable 2-period cycles. Since $x^{*}, y^{*}$ and $J\left(x^{*}, y^{*}\right)$ are continuous with respect to $\alpha$ and $d$, if $d$ is close to 
(a)

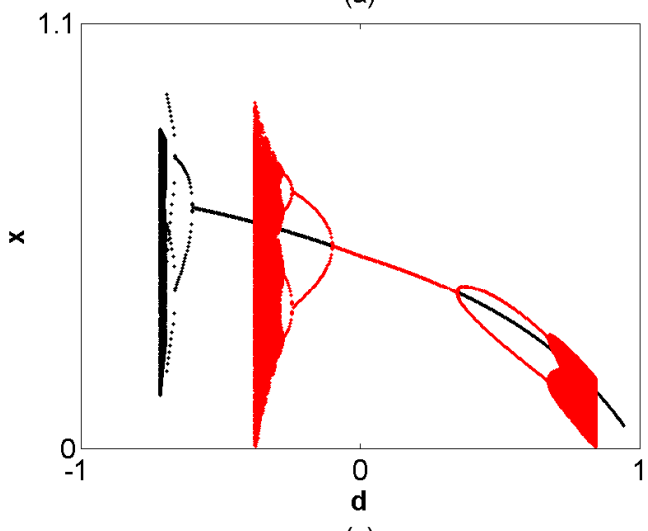

(c)

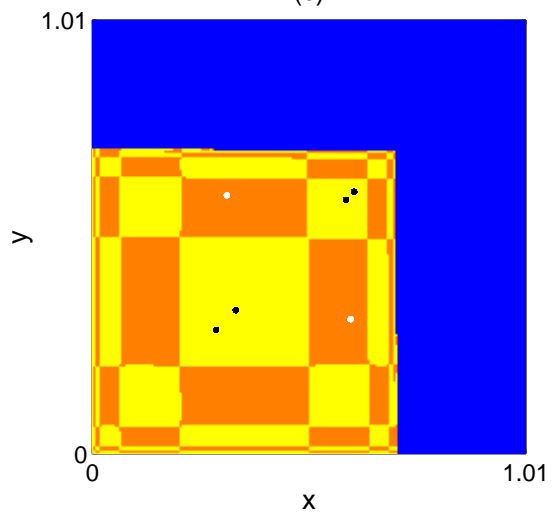

(b)

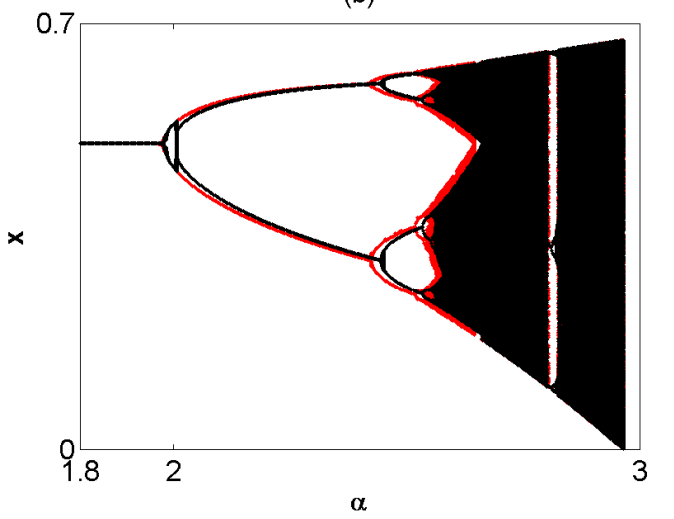

(d)

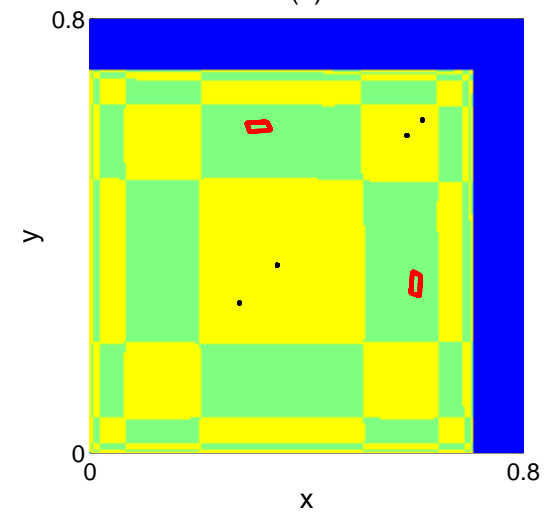

Figure 3: (a) One dimensional bifurcation diagrams of variable $x$ w.r.t. $d$ for $b_{1}=0.1$ and $b_{2}=0.2$ when $d<0$ or $b_{1}=-0.1$ and $b_{2}=-0.2$ when $d \geq 0$ and two different values of $\alpha: \alpha=1.8$ in the red diagram while $\alpha=0.8$ in the black diagram. (b) Parameter values: $d=-0.01, b_{1}=0.1$ and $b_{2}=0.2$. As $\alpha$ varies the bifurcation diagram in red is obtained for $x(0)=0.2$ and $y(0)=0.21$ while the bifurcation diagram in black is obtained if $x(0)=0.37$ and $y(0)=0.57$. (c) A 2-period cycle (white points) coexists with a 4-period cycle (black points) for $\alpha=2.44$ and the other parameters as in $(b)$. (d) A 4-period cycle (black points) coexists with two cyclic attracting invariant curves (red points) for $\alpha=2.46$ and the other parameters as in $(b)$. 
zero then $\lambda_{1}=-1$ or $\lambda_{2}=-1$ for two given values of $\alpha$ close to 2 , i.e. the flip bifurcation still occurs, $\forall b_{1}, b_{2}$. An interesting phenomenon can be observed in this case.

Let $d \rightarrow 0$ (that is, products tend to be independent between each other). Then system $T$ tends to a diagonal system where both equations are conjugated to the logistic map and the standard period doubling bifurcation is produced as $\alpha$ is varied. In addition, the eigenvalues $\lambda_{1}$ and $\lambda_{2}$ (associated to fixed point $E^{*}$ or to a periodic point), tends to be equal. For this reason, if at a given value of $\alpha=\bar{\alpha}$ a flip bifurcation occurs along one direction (for instance $\lambda_{1}=-1$ ) then the same flip bifurcation occurs along the other direction (for instance the one associated to $\lambda_{2}$ ) for a given value of $\alpha$ close to $\bar{\alpha}$ (to better understand this phenomenon, see Bischi \& Kopel, 2003). This fact can be clarified by looking at Figure $3(b)$, where two bifurcation diagrams with respect to $\alpha$ are plotted by fixing $d$ close to zero but choosing different suitable initial conditions. The red diagram is obtained by starting from an initial condition $(x(0), y(0))$ with $x(0)$ close to $y(0)$ (that is firms enter the market with similar prices), while the black diagram is plotted by choosing $x(0)$ far enough from $y(0)$ (i.e., initial prices substantially differ). It can be observed that the two diagrams are very similar: they exhibit the period doubling bifurcation of the logistic map if $\alpha$ is not too high (almost all trajectories are unfeasible if $\alpha$ is large enough, as is shown in part (iii) of Proposition 1). However, a particular phenomenon of multistability with the creation of several coexisting attracting cycles can be observed when the speed of adjustment of prices is relatively large. For instance, Figure $3(c)$ shows the existence of a locally stable 4-period cycle that coexists with a locally stable 2-period cycle when $\alpha=2.44$, while in panel $(d)$ the 4-period cycle coexists with two cyclical attracting closed invariant curves created around the unstable 2-period cycle (due to a Neimark-Sacker bifurcation) for $\alpha=2.46$. In both panels the blue region represents the unfeasible set, while the two basins of attraction are depicted with different colors. In the numerical simulations presented in Figure $3(c)$ and $(d)$, it can be observed that one attractor is close to the diagonal (the 4-period cycle). Anyway, initial conditions having $x(0)$ close to $y(0)$ can produce trajectories converging to a different attractor, since two attractors coexist and the related basins are complex. In fact, when multistability arises, i.e. several attractors coexist, each of which with its own basin of attraction, the selected long-term state becomes path dependent and the structure of the basins of different attractors becomes crucial for predicting the long-term outcome of the economic system. More in detail, if the basins of attraction are connected sets, then the final evolution of the system can be predicted, while if the basins are composed by several non-connected sets, as in Figure 3 $(c)$ and $(d)$, then it is possible that an initial condition taken close to an attractor will produce a trajectory converging to the other one.

This result has non negligible policy consequences, as it encompasses elements related to the degree of product differentiation and the speed of adjustment of prices. Specifically, if products are not too much substitutes or complements but prices are adjusted gradually by each manager, then entering the market prices close to each other or with significantly different ones does not matter for long-term performances of the economy, as trajectories tend to the same attractor. In contrast, problems of unpredictability can actually exist if for some reasons the speed of adjustment of prices becomes larger. In this case, in fact, there are coexisting attractors, each of which with its own basin of attraction. Of course, policies aiming at changing strategically the initial condition may produce unpleasant results in this case. For instance, if a manager decides to reduce the initial price of his own product to increase the quantity produced and get higher profits, the economy may actually end up with significantly different findings.

We now want to focus on the local stability of $E^{*}$ when manager types tend to some limiting 
cases, i.e. $b_{i} \rightarrow 0$ and/or $\left|b_{i}\right| \rightarrow 1, i=1$ or $i=2$. The following Proposition can be proved.

Proposition 6. Let $T$ be given by system (7).

(i) Assume $b_{i} \rightarrow 0, i=1,2$. Then if $\alpha \geq 2, E^{*}$ is locally unstable, while $\forall \alpha \in(0,2) E^{*}$ is locally stable iff $d \in\left(d_{-}, d_{+}\right)$, where $d_{-}=\frac{\alpha-2}{2} \in(-1,0)$ and $d_{+}=\min \left\{\frac{2-\alpha+\sqrt{(2-\alpha)^{2}+16(2-\alpha)}}{4}, 1\right\} \in$ $(0,1)$.

(ii) Assume $b_{i} \rightarrow 1^{-}$(resp. $\left.b_{i} \rightarrow-1^{+}\right), i=1,2$. Then if $\alpha \geq 2, E^{*}$ is locally unstable, while $\forall \alpha \in(0,2) E^{*}$ is locally stable iff $d \in\left(-\frac{2-\alpha}{2}, 0\right)$ (resp. $d \in\left(0, \frac{2-\alpha}{2}\right)$ ).

(iii) Assume $b_{i} \rightarrow 1^{-}$and $b_{j} \rightarrow 0^{+}, i, j=1,2 i \neq j$. Then if $\alpha \geq 2, E^{*}$ is locally unstable, while $\forall \alpha \in(0,2) E^{*}$ is locally stable iff $d \in\left(-\frac{2-\alpha}{2}, 0\right)$.

(iv) Assume $b_{i} \rightarrow-1^{+}$and $b_{j} \rightarrow 0^{-}, i, j=1,2 i \neq j$. Then if $\alpha \in I_{+}(0)$, where $I_{+}(0)$ is the right neighnorhood of $0, E^{*}$ is locally stable iff $\forall d \in(0,1)$.

Proof. (i) If $b_{i} \rightarrow 0, i=1,2$, then both $x^{*}$ and $y^{*}$ tend to $\frac{1-d}{2-d}$ while

$$
J\left(x^{*}, y^{*}\right) \rightarrow\left(\begin{array}{cc}
A & B \\
B & A
\end{array}\right), A=1-2 \frac{\alpha}{(1+d)(2-d)}, B=\frac{\alpha d}{(1+d)(2-d)} .
$$

As a consequence $\lambda_{1}\left(x^{*}, y^{*}\right) \rightarrow A-B=1-\alpha \frac{2+d}{(1+d)(2-d)}<1$ while $\lambda_{2}\left(x^{*}, y^{*}\right) \rightarrow A+B=$ $1-\alpha \frac{1}{1+d}<1$ which depends on $\alpha$ and $d$. We now distinguish between the cases of $d<0$ and $d>0$. If $d \in(-1,0)$ then $\frac{2+d}{2-d} \in\left(\frac{1}{3}, 1\right)$ and consequently $\lambda_{2}\left(x^{*}, y^{*}\right)<\lambda_{1}\left(x^{*}, y^{*}\right) \forall b_{i} \in I_{+}(0)$, $i=1,2$. Hence $\lambda_{2}\left(x^{*}, y^{*}\right)>-1$ iff $E^{*}$ is locally stable. From condition $A+B>-1$ it follows that if $\alpha \geq 2$ then $E^{*}$ is locally unstable while, if $\alpha \in(0,2)$, then simple computations show that $E^{*}$ is locally stable as long as $d \in\left(\frac{\alpha-2}{2}, 0\right)$.

Similarly, if $d \in(0,1)$ then $\frac{2+d}{2-d} \in(1,3)$ hence, $\forall b_{i} \in I_{-}(0), i=1,2, \lambda_{1}\left(x^{*}, y^{*}\right)<\lambda_{2}\left(x^{*}, y^{*}\right)$ and $\lambda_{1}\left(x^{*}, y^{*}\right)>-1$ iff $E^{*}$ is locally stable. It can be easily verified that if $\alpha \geq 2$ then $E^{*}$ is locally unstable while, if $\alpha \in(0,2)$, then $A-B>-1$ iff $-2 d^{2}+(2-\alpha) d+2(2-\alpha)>0$ thus reaching condition $d \in\left(0, d_{+}\right)$.

(ii) Following the same procedure used to prove part $(i)$, it can be easily verified that the following two cases may occur. If $b_{i} \rightarrow 1^{-}, i=1,2$, then both $\lambda_{1}\left(x^{*}, y^{*}\right)$ and $\lambda_{2}\left(x^{*}, y^{*}\right)$ tend to $1-\frac{\alpha}{1+d}<1$. Hence using condition $1-\frac{\alpha}{1+d}>-1$ together with $d<0$ the statement can be proved. Similarly, if $b_{i} \rightarrow-1^{+}, i=1,2$, then $\lambda_{1}\left(x^{*}, y^{*}\right) \rightarrow 1-\frac{\alpha}{1+d}<1$ while $\lambda_{2}\left(x^{*}, y^{*}\right) \rightarrow 1-\frac{\alpha}{1-d}<1$. By considering conditions $\lambda_{1}\left(x^{*}, y^{*}\right)>-1, \lambda_{2}\left(x^{*}, y^{*}\right)>-1$ and $d>0$ the statement is proved.

(iii) Similarly to the arguments used to prove part $(i)$, it can be observed that if, for instance, $b_{1} \rightarrow 1^{-}$and $b_{2} \rightarrow 0^{+}$then $\lambda_{1}\left(x^{*}, y^{*}\right) \rightarrow 1-\alpha \frac{1}{1+d}<1$ while $\lambda_{2}\left(x^{*}, y^{*}\right) \rightarrow 1-\alpha \frac{1}{1+d} \frac{2+d}{2}<1$. Hence $E^{*}$ is locally stable iff $1-\alpha \frac{1}{1+d}>-1$ that is $\alpha \in(0,2)$ and $d \in\left(-\frac{2-\alpha}{2}, 0\right)$.

(iv) Without loss of generality, let $b_{1} \rightarrow-1^{+}$and $b_{2} \rightarrow 0^{-}$, then $x^{*} \rightarrow \frac{1-d^{2}}{2-d^{2}}$ and $y^{*} \rightarrow \frac{(1-d)(2+d)}{2\left(2-d^{2}\right)}$ while

$$
J\left(x^{*}, y^{*}\right) \rightarrow\left(\begin{array}{cl}
1-\frac{2 \alpha}{2-d^{2}} & \frac{2 \alpha d}{2-d^{2}} \\
\frac{\alpha d(2+d)}{(1+d) 2\left(2-d^{2}\right)} & 1-\frac{\alpha(d+2)}{(1+d)\left(2-d^{2}\right)}
\end{array}\right) .
$$

Following Medio and Lines 2001, $E^{*}$ is locally stable if the following conditions in terms of trace $(t r)$ and determinant (det) of matrix $J\left(x^{*}, y^{*}\right)$ are fulfilled: 
(a) $\psi_{1}(\alpha, d)=1+\operatorname{tr}\left(J\left(x^{*}, y^{*}\right)\right)+\operatorname{det}\left(J\left(x^{*}, y^{*}\right)\right)>0$

(b) $\psi_{2}(\alpha, d)=1-\operatorname{tr}\left(J\left(x^{*}, y^{*}\right)\right)+\operatorname{det}\left(J\left(x^{*}, y^{*}\right)\right)>0$,

(c) $\psi_{3}(\alpha, d)=1-\operatorname{det}\left(J\left(x^{*}, y^{*}\right)\right)>0$.

After some algebra, it can be verified that:

(a) if $\alpha \in I_{+}(0)$ then $\psi_{1}(\alpha, d)=4-\alpha \frac{2(3 d+4)-\alpha(2+d)}{\left(2-d^{2}\right)(1+d)}>0 \forall d \in(0,1)$,

(b) $\psi_{2}(\alpha, d)=\frac{\alpha^{2}(2+d)}{\left(2-d^{2}\right)(1+d)}>0, \forall \alpha>0$ and $\forall d \in(0,1)$,

(c) if $\alpha \in(0,2)$ then $\psi_{3}(\alpha, d)=\alpha \frac{(3 d+4)-\alpha(2+d)}{(1+d)\left(2-d^{2}\right)}>0 \forall d \in(0,1)$.

Then the statement is proved.

According to Proposition $6(i)$, if managers attitudes are close to zero, the Nash equilibrium is locally stable iff $\alpha$ is not too high $(\alpha<2)$ and $d$ belongs to an intermediate range of values $\left(d \in\left(d_{-}, d_{+}\right)\right)$, as confirmed in Figures $3(a)$ and $(b)$. In addition, the following evidences emerge. First, if $\alpha>2$ then the Nash equilibrium is locally unstable for all $d \in(-1,1)$ (see Figure $4(a)$ ) and multistability may arise (see Figure $4(b)$, where three coexisting attractors are depicted) so that the problem of path dependence emerges. Second, if the degree of horizontal differentiation between products tends to \pm 1 (products tend to be perfect substitutes or perfect complements), the Nash equilibrium is locally unstable $\forall \alpha$ (confirming the results proved in parts $(i)$ and $(i i)$ of Proposition 1). Third, if $\alpha \in(0,2)$ and products tend to be sufficiently complements or substitutes (by crossing $d_{-}$or $d_{+} E^{*}$ becomes a saddle point in which a 2-period cycle is created) then, if $d$ further increases or decreases, more complex attractors may emerge, as depicted in Figure $4(c)$ and $(d)$. Other relevant economic consequences can be inferred in this case. Indeed, if $\alpha$ sufficiently large or the modulus of degree of product differentiation is large, then there exists a chaotic attractor and also multistability occurs. This seems to suggest that advertising policies aiming at reducing the degree of substitutability or complementarity of products (or policies that tend to reduce the speed of adjustment of prices) are able to smooth endogenous fluctuations by also allow managers to predict the long-term behaviour of the economy.

Proposition 6 (ii) concerns the limiting cases in which managers' attitudes are either close to -1 and products are substitutes or close to +1 and products are complements. Also in this case it is confirmed that the Nash equilibrium is locally stable iff $\alpha$ is not too high and $d$ belongs to an intermediate range of value (indeed, several numerical experiments show that in this case $E^{*}$ is globally stable). Furthermore, as the eigenvalues associated to the fixed point or to a periodic point tend to be equal, then a phenomenon similar to the one described for the case $d \rightarrow 0$ emerges. To better clarify this finding, let $b_{1}$ and $b_{2}$ be close to 1 . Then, if $\alpha=1$ the fixed point $E^{*}$ is locally stable as long as $d \in(-0.5,0)$, while at $d \simeq-0.5$ a flip bifurcation occurs and two locally stable 2-period cycles are created almost simultaneously. Again, as $d$ decreases they become unstable and two locally stable 4-period cycles are created (see Figure $5(a)$ ) and so on, providing that also in this cases coexisting attractors are exhibited until almost all initial conditions become unfeasible. A similar behaviour occurs also in the other limiting case, i.e. $b_{1}$ and $b_{2}$ are close to -1 . From an economic point of view, this result suggests that as long as the degrees of competition or cooperation of both manager sharply increase, the absolute value of marginal profits tends to increase so that managers overreact and set prices either at too large or too small values (even if $\alpha$ and $d$ are not too high) thus causing fluctuations and multistability. 
(a)

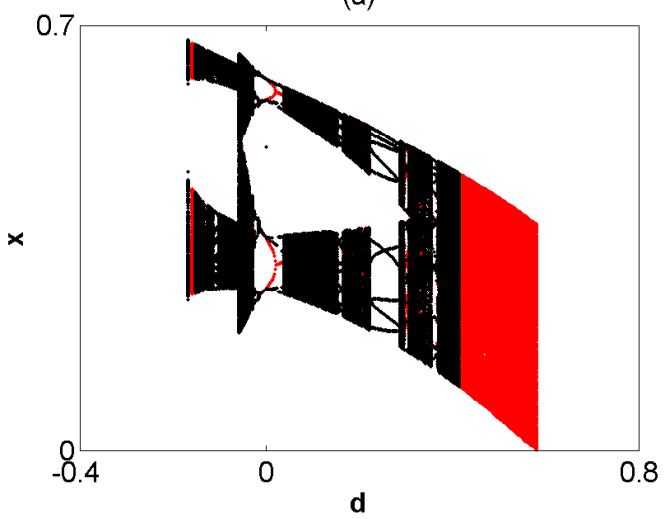

(c)

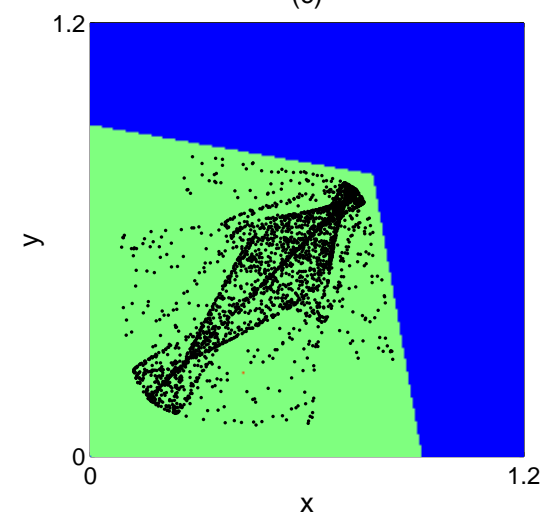

(b)

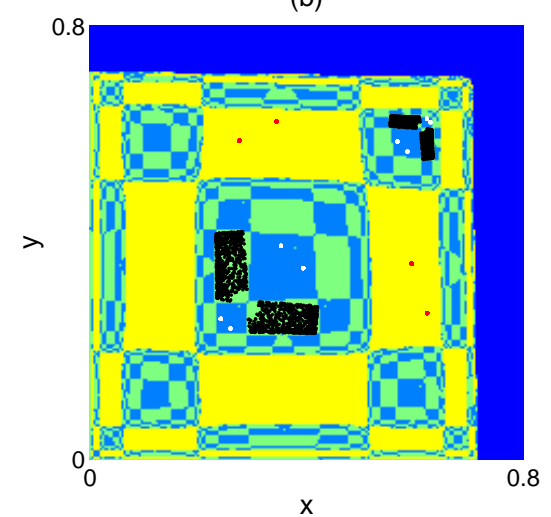

(d)

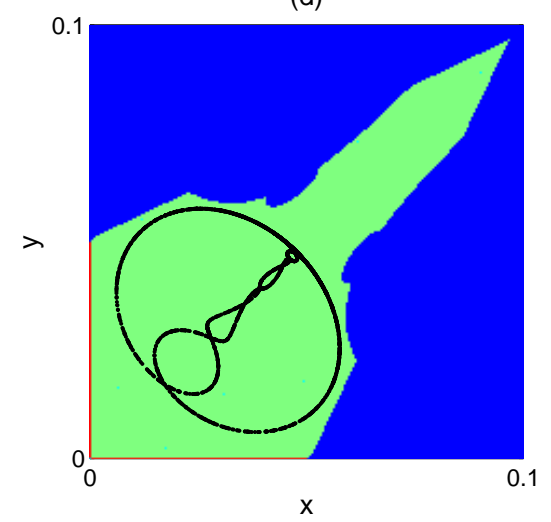

Figure 4: (a) One dimensional bifurcation diagrams of variable $x$ w.r.t. $d$ for $b_{1}=0.001$ and $b_{2}=0.002$, when $d<0$, or $b_{1}=-0.001$ and $b_{2}=-0.002$, when $d \geq 0$, and $\alpha=2.5$; two different initial condition are considered: $(0.2,0.2)$ in the red diagram and $(0.5,0.2)$ in the black diagram. $(b)$ A 4-pieces complex attractor (black points) coexists with a 4-period cycle (red points) and a 8-period cycle 8black points) for $d=-0.03$ and the other parameters as in $(a)$. (c) Complex attractor for $\alpha=1.8, d=-0.35$ and the other parameters as in $(a)$. (d) Complex attractor for $\alpha=1.9, d=0.95$ and the other parameters as in $(a)$. 
(a)

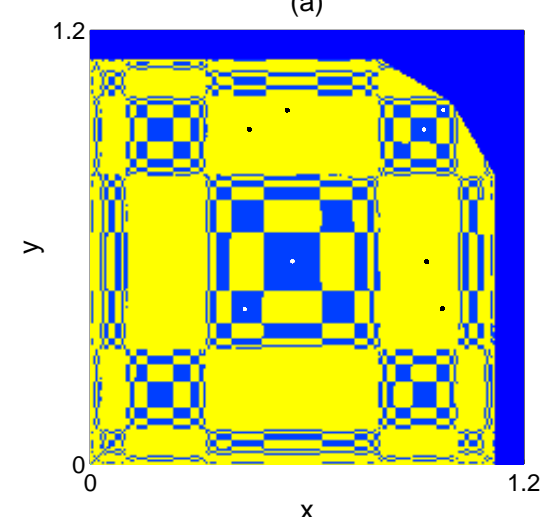

(c)

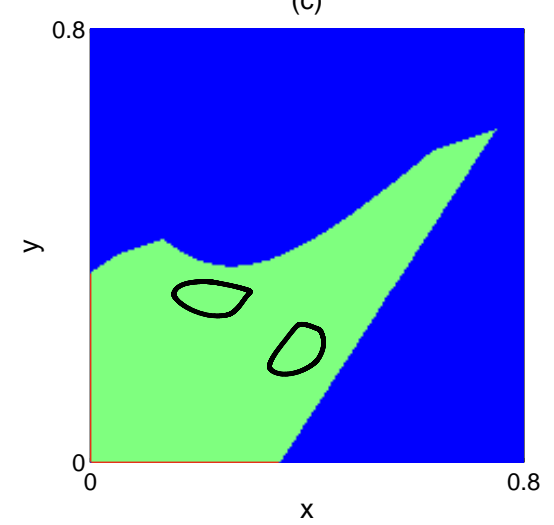

(b)

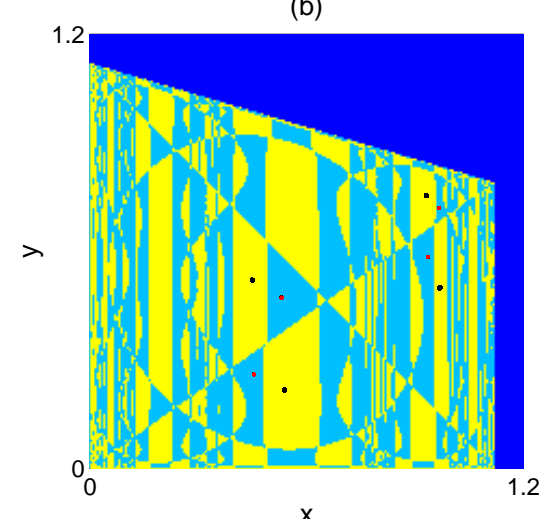

(d)

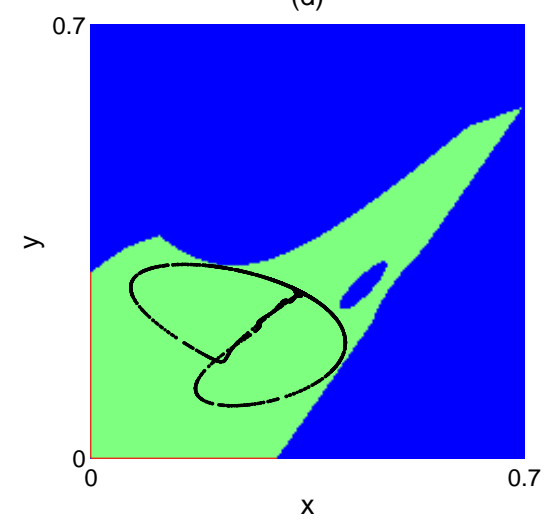

Figure 5: (a) Two coexisting attracting 4-period cycles are exhibited for $b_{1}=0.99, b_{2}=0.999, \alpha=1$ and $d=-0.6$. (b) Two coexisting attracting 4-period cycles are exhibited for $b_{1}=0.99, b_{2}=0.01$, $\alpha=1$ and $d=-0.595$. (c) Two two cyclic attracting closed invariant curves are presented for $b_{1}=-0.99, b_{2}=-0.01, \alpha=1.5$ and $d=0.65$. (d) One piece chaotic attractor for $b_{1}=-0.99$, $b_{2}=-0.01, \alpha=1.5$ and $d=0.7$. 
Finally, Proposition $6(i i i)$ and $(i v)$ is related to the case in which managers attitudes move in the opposite way, and heterogeneity becomes prominent. To this purpose, let us consider first the case in which products are complements (so that $b_{1}$ and $b_{2}$ are positive). Then, if one type tends to be very high and the other type is very small (one manager behaves more aggressively than the rival does), the condition for the local stability of the Nash equilibrium is the same as in previous case (ii), i.e. both $\alpha$ and $d$ must be not too small. Again, in this case, the stability of $E^{*}$ is global. However, differently from the previous case, if $b_{1}$ and $b_{2}$ tend towards different values, then the attractor existing close to the diagonal is lost and the basins structure appear different (and more complex). In Figure $5(b)$ the case with substantially different manager types is presented: as in panel $(a)$, two coexisting 4-period cycles are depicted. On the one hand, in panel $(b)$ both attractors are characterised by different behaviour between firms, and the corresponding basins are more complex. On the other hand, if products are substitutes and managers attitudes tend to opposite limiting values (one manager behaves more cooperatively than the rival does), then only a sufficient condition for the local stability of the Nash equilibrium can be given (i.e. $\alpha$ low enough). If $\alpha$ is not too small, for instance $\alpha=1.5$, then it can be observed that $E^{*}$ is globally stable if $d$ is not too high, while at $d \simeq 0.42$ the fixed point $E^{*}$ becomes unstable and a globally stable 2-period cycle appears. Such an attractor persists until, if $d$ further increases, two cyclical attracting closed invariant curves are created - due to a Neimark-Sacker bifurcation occurring at $d \simeq 0.626$ - around the unstable 2-period cycle (see Figure $5(c)$ ). For a larger value of $d$ the attractor consists of a onepiece chaotic attractor (see Figure $5(d)$ ) and a hole of the unfeasible set appears inside $D$. This result seems to suggest that in addition to endogenous fluctuations, if managers' behaviours substantially differ (for example, one manager strongly competes and the other does not) the structure of the basin of attraction becomes more complex, because the weights of managers' attitudes enter differently in their own marginal bonuses, so that unpredictability depending on initial conditions is a plausible scenario.

\section{THE SYMMETRIC CASE OF EQUAL MANAGER AT- TITUDE: SYNCHRONISATION AND INTERMITTENCY}

We now consider the particular case obtained under the assumption $b_{1}=b_{2}=b$. In this case, the two managers behave in the same way and system $T$ in (7) takes the following form:

$$
T_{b}:\left\{\begin{array}{l}
x^{\prime}=x\left[1+\alpha\left(\frac{(1-d)-2 x+d(1-b) y}{1-d^{2}}\right)\right] \\
y^{\prime}=y\left[1+\alpha\left(\frac{(1-d)-2 y+d(1-b) x}{1-d^{2}}\right)\right],
\end{array},\right.
$$

which is a symmetric system in the sense that $T_{b}$ remains the same under the exchange of players, that is $T_{b} \circ S=S \circ T_{b}$, where $S:(x, y) \rightarrow(y, x)$ is the reflection through the diagonal $\left\{(x, y) \in \mathbb{R}_{+}^{2}: x=y\right\}$. The dynamics of $T_{b}$ can be studied by following the same arguments used in Fanti et al. 2013, so that in what follows we only recall some evidences that are useful to explain the new economic results.

Map $T_{b}$ admits a unique interior fixed point given by

$$
E_{b}^{*}=\left(x_{b}^{*}, x_{b}^{*}\right), x_{b}^{*}=\frac{(1-d)}{2-d(1-b)},
$$

and the set

$$
\Delta=\{(x, y) \in D: x \in[0,1], y=x\}
$$


is invariant for $T_{b}$. In addition, the dynamics of $T_{b}$ on $\Delta$ (called synchronised trajectories) are governed by the one-dimensional map

$$
\phi(x)=x\left[1+\alpha \frac{1-d+[d(1-b)-2] x}{1-d^{2}}\right]
$$

which is topologically conjugated to the standard logistic map $\omega^{\prime}=\mu \omega(1-\omega)$ and consequently, the dynamics generated by map $T_{b}$ on the diagonal are completely known.

A preliminary important observation is that, differently form Fanti et al. (2014), since $\mu=1+\frac{\alpha}{1+d}$ does not depend on $b$, then the qualitative long-term synchronised dynamics does not depend on manager's attitude, which indeed affects the quantitative dynamics.

We present the long-term synchronised dynamics qualitatively in the cycle cartogram depicted in Figure $6(a)$. It shows a two-parameter bifurcation diagram where each color describes a long-term behavior for a given combination of $d$ and $\alpha$. Cycles of different order $i$ (i.e. $C i$ ) are exhibited and other more complex dynamics can be obtained. Notice that the $d$-interval associated with the occurrence of cycles or complex dynamics moves to the right as $\alpha$ increases. There is an interesting relationship between $\alpha$ and $d$ in this case that can be noticed. For small values of $\alpha$ complex dynamics are possible only when prices are strategic substitutes (products are complements). In this kind of models (Bischi et al., 1998) complexity arises when the speed of adjustment of players (ceteris paribus) increases. However, in this context an increase in $\alpha$ is a sources of complex dynamic only whether prices tend to become strategic complements (products are substitutes). In the former case, profits are smaller so that the marginal bonus of managers is relatively small because $d$ tends to become high in modulus. In the latter case, both managers want to benefit from cooperation and then overreact by increasing the marginal bonus too much. The black bifurcation curves depicted in the figure correspond to the set of points such that $\mu=3$ (curve $C$ ) and $\mu=4$ (curve $C^{\prime}$ ) and represent two bifurcation values of the logistic map. In particular, the set $\{(d, \alpha): 1<\mu<3\}$, given by points located below curve $C$, are such that the fixed point is stable (i.e. the synchronised trajectories converge to the Nash equilibrium), while the set $\{(d, \alpha): \mu>4\}$, given by the points located above curve $C^{\prime}$, characterises the parameter region such that almost all synchronised trajectories are unfeasible. The region between curves $C$ and $C^{\prime}$ is associated to the standard period doubling bifurcation cascade occurring as $d$ decreases for a given (not too high) value of $\alpha$ (see Figure $6(b)$, where we have considered two different values of $b$ ). Differently from Fanti et al. (2014) $b$ does not affect the qualitative dynamics of synchronised trajectories (the bifurcation values of $d$ are the same) but only the quantitative dynamics (the asymptotic states are smaller if $b$ is higher). Synchronisation is certainly an interesting phenomenon as it implies that players behave in the same way in the long term. This is important especially in the case prices are strategic complements as managers may gain by sharing higher profitability.

If $A \subseteq \Delta$ is an attracting set of $\phi$ then in order to study the stability of $A$ for $T_{b}$ we have to consider the transverse stability, as in Fanti et al. $(2014,2015)$ for the case of market share bonus, so that we now underline the main results related to the present model in comparison with the previous ones, and we refer to them for further details.

Notice that in the symmetric case of equal managers attitude, the Jacobian matrix in (8) evaluated at a point on $\Delta$ is given by

$$
\begin{gathered}
J_{b}(x, x)= \\
\left(\begin{array}{cc}
J_{1}(x) & J_{2}(x) \\
J_{2}(x) & J_{1}(x)
\end{array}\right)
\end{gathered}
$$




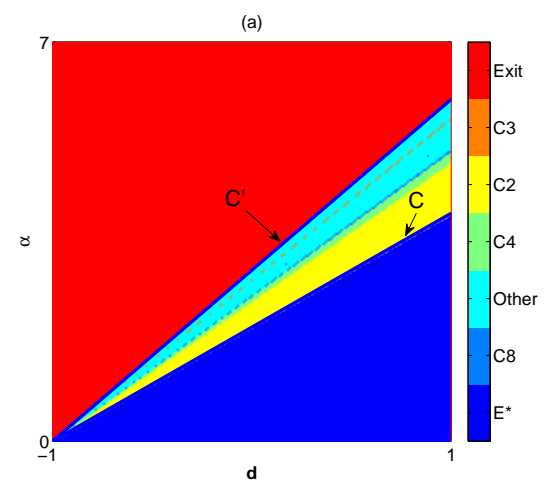

(c)

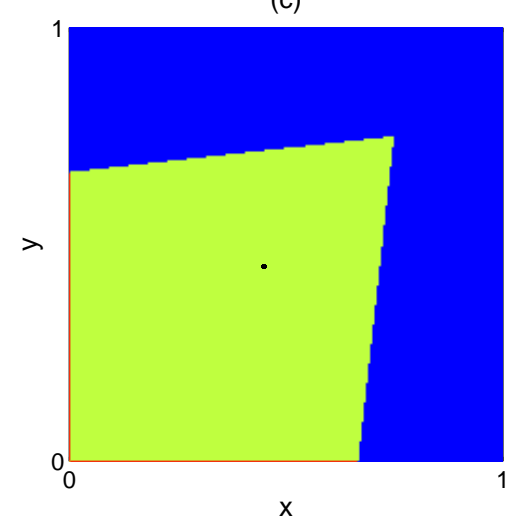

(b)

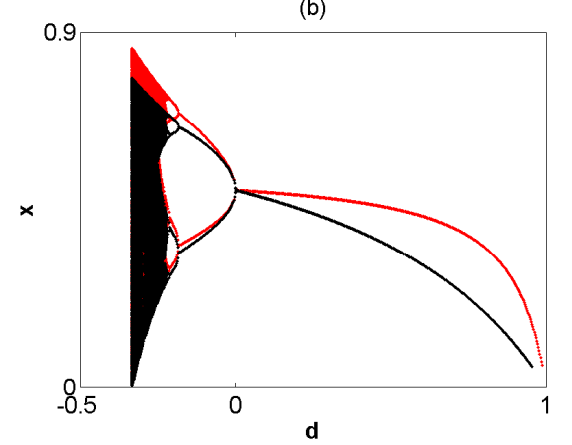

(d)

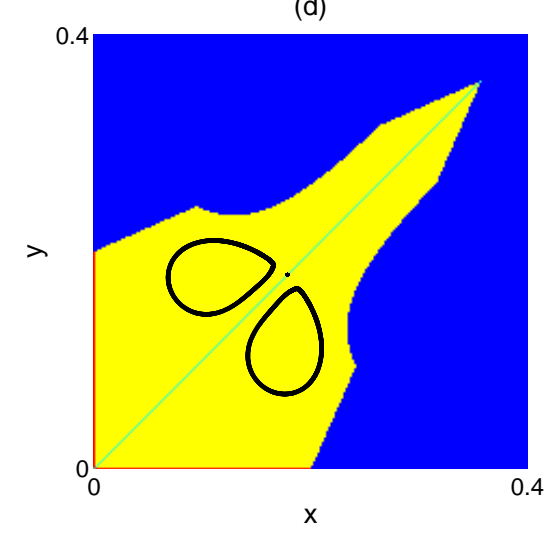

Figure 6: (a) Two dimensional bifurcation diagram of map $\phi$ in the plane $(d, \alpha)$ for any given value of $b$. (b) One dimensional bifurcation diagram of map $\phi$ w.r.t. $d$ for $\alpha=2$ : if $|b|=0.8$ the red diagram is obtained while if $|b|=0.2$ the black one is produced. $(c)$ The Nash equlibrium and its basin for $\alpha=1.8, d=0.2$ and $b=-0.1$. (d) For $d=0.8$ and the other parameters as in (c) two coexisting attractors are presented and synchronization does not occur. 
where

$$
J_{1}(x)=1+\frac{\alpha}{1+d}-\frac{\alpha}{1-d^{2}}(4-d(1-b)) x,
$$

and

$$
J_{2}(x)=\frac{\alpha d(1-b)}{1-d^{2}} x .
$$

If the attractor $A$ on the diagonal is given by $E_{b}^{*}$, the phenomenon of synchronisation may easily be investigated. In fact, the eigenvalues of $J_{b}(x, x)$ evaluated at the fixed point are both real and they are given by:

$$
\lambda_{b \|}\left(E_{b}^{*}\right)=J_{1}\left(x_{b}^{*}\right)+J_{2}\left(x_{b}^{*}\right)=1-\frac{\alpha}{1+d}<1,
$$

and

$$
\lambda_{b \perp}\left(E_{b}^{*}\right)=J_{1}\left(x_{b}^{*}\right)-J_{2}\left(x_{b}^{*}\right)=1-\frac{\alpha}{1+d} \frac{2+d(1-b)}{2-d(1-b)}<1,
$$

while the corresponding eigenvectors are respectively given by $\underline{\mathrm{v}}_{b \|}=(1,1)$ and $\underline{\mathrm{v}}_{b \perp}=(1,-1)$.

The interior fixed point $E_{b}^{*}$ can be attracting for suitable values of the parameters such that $\lambda_{\|}\left(x_{b}^{*}\right)>-1$ and $\lambda_{\perp}\left(x_{b}^{*}\right)>-1$. The following Proposition can then be proved.

Proposition 7. Assume that $E_{b}^{*}$ is the attractor of $T_{b}$ on $\Delta$. Then: (i) if $d<0, E_{b}^{*}$ is also transversely stable; (ii) if $d>0, E_{b}^{*}$ can be transversely stable or transversely unstable.

Proof. If $d<0$ (resp. $>$ ) then $\frac{2+d(1-b)}{2-d(1-b)}<1$ (resp. $>$ ) and consequently $\lambda_{\|}\left(x_{b}^{*}\right)<\lambda_{\perp}\left(x_{b}^{*}\right)$ (resp. $>$ ). Hence, if $d<0$ then $\lambda_{\|}\left(x_{b}^{*}\right)>-1$ implies $\lambda_{\perp}\left(x_{b}^{*}\right)>-1$ while if $d>0$ then $\lambda_{\|}\left(x_{b}^{*}\right)>-1$ does not imply $\lambda_{\perp}\left(x_{b}^{*}\right)>-1$.

According to the previous statement, if products are complements and synchronised trajectories converge to the Nash equilibrium, then synchronisation takes place, i.e. firms starting from different initial conditions may coordinate in the long term, as for the Bertrand duopoly with market share bonus studied in Fanti et al. (2015).

On the other hand, synchronisation toward the Nash equilibrium may or may not occur if products are substitutes. More precisely, by looking at the proof of Proposition 7, it can be observed that if $d>0$ and $E_{b}^{*}$ is transversely stable then it is also locally stable, while the opposite is not true in general. For this reason, if $E_{b}^{*}$ is locally stable and products are substitutes (i.e. $d>0$ ) then the fixed point becomes a saddle point by loosing its transverse stability as $d$ increases, so that trajectories do not synchronise (see Fanti et al., 2014). This result can be clarified by comparing panels $(c)$ and $(d)$ in Figure 6 . In fact, $E_{b}^{*}$ is globally stable for $d=0.2$ and synchronisation occurs; if $d$ increases then $\lambda_{\perp}\left(x_{b}^{*}\right)$ decreases so that it can cross the value -1 . Then synchronised trajectories still converge to the Nash equilibrium while synchronisation is avoided, as a locally stable 2-period cycle is created out of the diagonal. In Figure $6(d)$ shows (for a higher value of $d$ ) that the attractor outside the diagonal is comprised of two cyclical closed invariant curves created via a Neimark-Sacker bifurcation around the unstable 2-period cycle, and synchronisation does not take place.

Consider now the case in which $A$ is a $m$-cycle. Then, similarly to what happens for the fixed point, several numerical computations show that if $A$ is a $m$-cycle for $d>0$, then the $m$-cycle loses firstly its transverse stability as $d$ increases. Therefore, synchronisation do not occur. On the other hand, synchronisation is likely to emerge if products are complements (i.e., prices are strategic substitutes). Therefore, managers may avoid to reduce profitability too much through cooperation also when then behave competitively.

The same evidence holds also if $A$ is a chaotic attractor on $\Delta$, as it can be ascertained by considering the transverse Lyapunov exponent and the natural transverse Lyapunov exponent associated 
with $A$ (for further details see Bischi et al., 1998 and Bischi \& Gardini, 2000). The study follows the same steps presented in Fanti et al. (2013, 2015) to which we refer for more details. However, differently from previous studies in this work we stress the importance of the role played by parameter $b$ in a model with relative profit delegation. The manager attitude value does not affect the qualitative long-tern synchronised dynamics. Indeed, it affects the transverse stability of the $m$-cycle or the transverse stability associated with the more complex attractor on $\Delta$. This means that, for given fixed values of $\alpha$ and $d<0$, synchronised trajectories converge to $A$ on $\Delta$ for any $b$, but synchronisation may or may not occur depending on the value of $b$.

This fact can be clarified by looking at Figure 7. In panels $(a)$ and (b) the values of $\alpha$ and $d$ have been fixed and the attractor $A$ on the diagonal consists of a 8-period cycle. However, two different managers attitudes are considered, and it can be observed that, by changing $b$ both the structure of the attractor existing out of the diagonal and the occurrence of synchronisation differ: in panel (a) the 8-period cycle coexists with a complex attractor and synchronisation does not occur, while in panel $(b)$ the 8-period cycle coexists with a 2-period cycle and synchronisation emerges.

One may be interested in knowing if there exists a relationship between the occurrence of synchronisation and the size of parameter $b$. A unique relationship cannot actually be found. In fact, differently from the results shown in panels $(a)$ and $(b)$ of Figure 7 , by looking at panels $(c)$ and $(d)$ it can be observed that the synchronisation phenomenon is associated with a smaller values of $b$. Furthermore, it can also be noticed that in cases $(c)$ and $(d)$ the attractor on the diagonal and the coexisting attractor out of the diagonal are both complex. However, the two basin structures appear quite different. In panel $(c)$ the basin structure is simple, synchronisation does not occur and the qualitative long-term dynamics can be predicted. In contrast, panel $(d)$ shows that the basins of attraction are complex thus giving rise to problems of unpredictability of the final outcome of the economy, as the final evolution of the economy is path dependent (slightly different initial conditions may lead to distinct qualitative long-term evolution and the occurrence of synchronisation cannot be predicted). In addition, in the case presented in panel $(d)$, transversely repelling trajectories can be re-injected toward $\Delta$ so that their behavior is characterised by some bursts far from the diagonal, before the synchronisation or before converging to a different attractor. This situation is called onoff intermittency (for more details see Fanti et al., 2013, 2015). The synchronisation phenomenon is associated with values of $b$ close to an intermediate range. Managers, therefore, may coordinate in the long-term (synchronisation) when prices are strategic substitutes only whether they do not behave too much aggressively.

\section{CONCLUSIONS}

This article has taken a dynamic view of the behaviour of managerial firms in a repeated duopoly with price competition, horizontal differentiation and relative performance. As pointed out by Miller \& Pazgal (2002, p. 61): "There is significant evidence that managers do, in fact, care about relative performance. This evidence comes from areas such as psychology, empirical economics, and the popular management literature" (for empirical support see Gibbons \& Murphy, 1990 and Janakiraman et al., 1992). By following the tradition of Bischi et al. (1998), this article has stressed the importance of limited information and behavioural heterogeneity as sources of endogenous fluctuations and complex dynamics in a duopoly with managerial firms. Indeed, markets today are subject to (sometimes markedly significant) changes in consumers' tastes and preferences, so that managers in oligopoly firms may not be able to get enough information to know the rival's atti- 
(a)

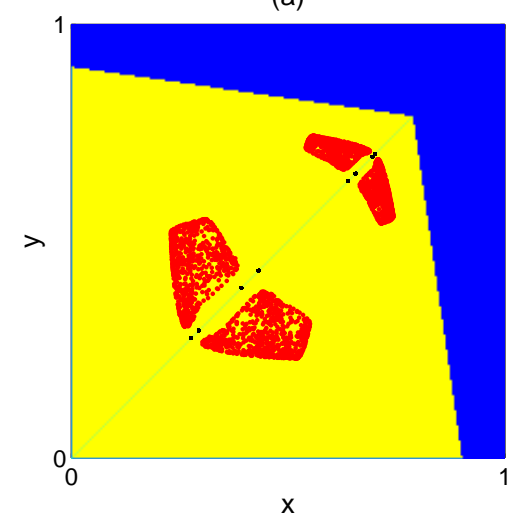

(c)

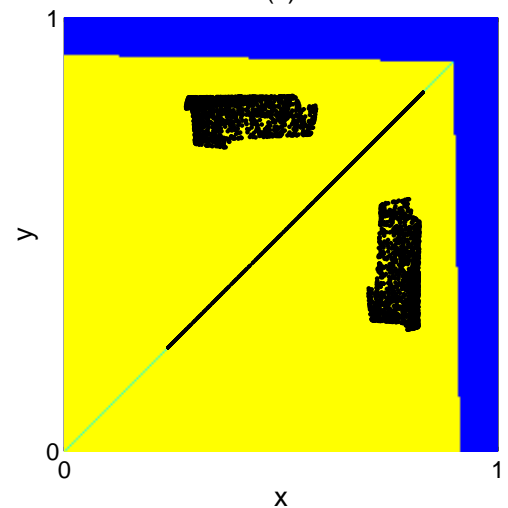

(b)

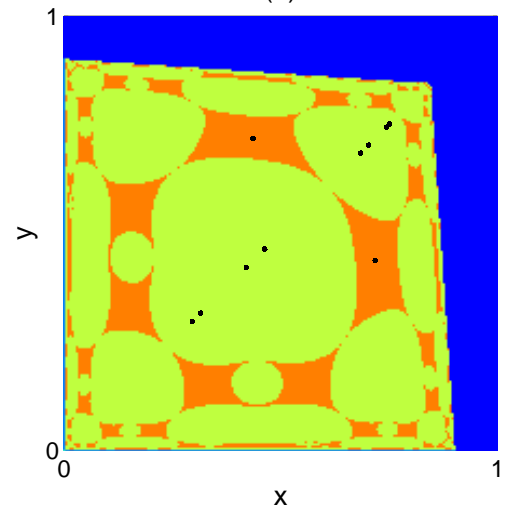

(d)

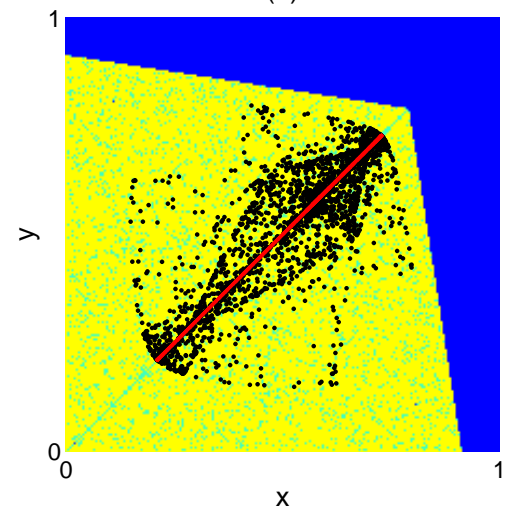

Figure 7: $\alpha=1.8$. ( $a$ ) If $d=-0.296$ and $b=0.03$ synchronization does not emerge; $(b)$ if $d=-0.296$ and $b=0.55$ synchronization occurs. (c) If $d=-0.33$ and $b=0.9$ synchronization does not emerge; $(d)$ if $d=-0.33$ and $b=0.06$ synchronization occurs. 
tude. In this context, we have found that the degree of product differentiation (that weights how consumers perceive products as being differentiated between each other) and managers' attitudes dramatically affect long-term dynamic outcomes. This holds when prices are strategic substitutes or strategic complements. In the former case, managerial selection process reduces competition and the outcome becomes more cooperative. In the latter case, managerial selection process increases the competitiveness of the firms. In both case, the behavioural rule that drives managers in setting the price from one period to the subsequent one, in fact, depends on the marginal bonus of the manager that is in turn affected by either variables (the product differentiation parameter and managers' attitude or type). If the marginal bonus is too large or too small, managers may overreact by favouring endogenous fluctuations. Specifically, we have found that if managers' behaviour in both firms is substantially different (for example, the manager hired in firm 1 competes aggressively but the manager hired in firm 2 does not) chaotic attractors, complex basins of attraction and multistability may actually occur. This gives rise to problems of unpredictability depending on initial conditions (i.e., the couple of prices chosen by managers to enter the market). Therefore, polices aiming at changing the degree of product differentiation (e.g., advertising polices), that also affect the attitude of managers to cooperate or compete, may become relevant as they may change the long-term scenario of the economy. With this regard, in the case of homogeneous attitude, managers may coordinate their behaviour (synchronisation) also when prices are strategic substitutes if they do not behave too much aggressively.

A possible extension of the present article is that of studying heterogeneous behaviour of players in models of Bertrand rivalry by considering one player with limited information and Local Monopolistic Approximation (as in Bischi et al., 2007; Cavalli and Naimzada, 2014; Cavalli et al., 2015) and the other player with full information and naïve expectations as in Puu (1991). This because the use of the Local Monopolistic Approximation rule has not yet been considered in price competition settings.

Acknowledgements: The authors gratefully acknowledge an Anonymous reviewer for valuable comments and suggestions on an earlier draft. The usual disclaimer applies.

\section{References}

[1] R. Abraham, L. Gardini, C. Mira, Chaos in Discrete Dynamical Systems. A Visual Introduction In Two Dimension. Springer-Verlag, Berlin (1997).

[2] R.K. Aggarwal, A.A. Samwick, "Executive compensation, strategic competition, and relative performance evaluation: theory and evidence," J. Financ. 54, 1999-2043 (1999).

[3] A. Agliari, C. Chiarella, L. Gardini, "A re-evaluation of adaptive expectations in light of global nonlinear dynamic analysis," J. Econ. Behav. Organ. 60, 526-552 (2006).

[4] J.R. Barro, R.J. Barro, "Pay, performance, and turnover of bank CEOs," J. Labor Econ. 8, 448-481 (1990).

[5] G.I. Bischi, L. Gardini, "Global properties of symmetric competition models with riddling and blowout phenomena," Discrete Dyn. Nat. Soc. 5, 149-160 (2000). 
[6] G.I. Bischi, M. Kopel, "Multistability and path dependence in a dynamic brand competition model," Chaos Soliton. Fract. 18, 561-576 (2003).

[7] G.I. Bischi, M. Gallegati, A.K. Naimzada, "Symmetry-breaking bifurcations and representative firm in dynamic duopoly games," Ann. Oper. Res. 89, 253-272 (1999).

[8] G.I. Bischi, C. Mammana, L. Gardini, "Multistability and cyclic attractors in duopoly games," Chaos Soliton. Fract. 11, 543-564 (2000).

[9] G.I. Bischi, A.K. Naimzada, L. Sbragia, "Oligopoly games with Local Monopolistic Approximation," J. Econ. Behav. Organ. 62, 371-388 (2007).

[10] G.I. Bischi, L. Stefanini, L. Gardini, "Synchronization, intermittency and critical curves in a duopoly game," Math. Comput. Simulat. 44, 559-585 (1998).

[11] S. Brianzoni, L. Gori, E. Michetti, "Dynamics of a Bertrand duopoly with differentiated products and nonlinear costs: analysis, comparisons and new evidences," Chaos Soliton. Fract. 79, 191-203 (2015).

[12] S. Brianzoni, C. Mammana, E. Michetti, "Non-linear dynamics in a business-cycle model with logistic population growth," Chaos Soliton. Fract. 40, 717-730 (2009).

[13] F. Cavalli, A.K. Naimzada, "A Cournot duopoly game with heterogeneous players: nonlinear dynamics of the gradient rule versus local monopolistic approach," Appl. Math. Comput. 249, $382-388$ (2014).

[14] F. Cavalli, A.K. Naimzada, F. Tramontana, "Nonlinear dynamics and global analysis of a heterogeneous Cournot duopoly with a local monopolistic approach versus a gradient rule with endogenous reactivity," Commun. Nonlinear Sci. Numer. Simulat. 23, 245-262 (2015).

[15] E.F. Fama, M.C. Jensen, "Separation of ownership and control," J. Law Econ. 26, 301-325 (1983).

[16] L. Fanti, L. Gori, M. Sodini, "Nonlinear dynamics in a Cournot duopoly with relative profit delegation," Chaos Soliton. Fract. 45, 1469-1478 (2012).

[17] L. Fanti, L. Gori, C. Mammana, E. Michetti, "The dynamics of a Bertrand duopoly with differentiated products: synchronization, intermittency and global dynamics," Chaos Soliton. Fract. 52, 73-86 (2013).

[18] L. Fanti, L. Gori, C. Mammana, E. Michetti, "Local and global dynamics in a duopoly with price competition and market share delegation," Chaos Soliton. Fract. 69, 253-270 (2014).

[19] L. Fanti, L. Gori, C. Mammana, E. Michetti, "Market share delegation in a Bertrand duopoly: synchronisation and multistability. Discrete Dyn. Nat. Soc. 2015, Article ID 394810 (2015).

[20] C. Fershtman, "Managerial incentives as a strategic variable in duopolistic environment," Int. J. Ind. Organ. 3, 245-253 (1985).

[21] C. Fershtman, K. Judd, "Equilibrium incentives in oligopoly," Am. Econ. Rev. 77, 927-940 (1987). 
[22] R. Gibbons, K.J. Murphy, "Relative performance evaluation for chief executive officers," Ind. Labor Relat. Rev. 43, 30S-51S (1990).

[23] L. Gori, L. Guerrini, M. Sodini, "A continuous time Cournot duopoly with delays," Chaos Soliton. Fract. 79, 166-177 (2015a).

[24] L. Gori, M. Sodini, L. Fanti, "A nonlinear Cournot duopoly with advertising," Chaos Soliton. Fract. 79, 178-190 (2015b).

[25] J. Häckner, "A note on price and quantity competition in differentiated oligopolies," J. Econ. Theory 93, 233-239 (2000).

[26] S. Janakiraman, R. Lambert, D. Larcker, "An empirical investigation of the relative performance evaluation hypothesis," J. Account. Res. 30, 53-69 (1992).

[27] T. Jansen, A. van Lier, A. van Witteloostuijn, "A Note on strategic delegation: the market share case," Int. J. Ind. Organ. 25, 531-539 (2007).

[28] T. Jansen, A. van Lier, A. van Witteloostuijn, "On the impact of managerial bonus systems on firm profit and market competition: the cases of pure profit, sales, market share and relative profits compared," Manage. Decis. Econ. 30, 141-153 (2009).

[29] M. Kopel, "Simple and complex adjustment dynamics in Cournot duopoly models," Chaos Soliton. Fract. 7, 2031-2048 (1996).

[30] M. Kopel, L. Lambertini, "On price competition with market share delegation contracts," Manage. Decis. Econ. 34, 40-43 (2013).

[31] D. Léonard, K. Nishimura, "Nonlinear dynamics in the Cournot model without full information," Ann. Oper. Res. 89, 165-173 (1999).

[32] A. Matsumoto, F. Szidarovszky, "Nonlinear delay monopoly with bounded rationality," Chaos Soliton. Fract. 45, 507-519 (2012).

[33] A. Matsumoto, F. Szidarovszky, "Discrete and continuous dynamics in nonlinear monopolies," Appl. Math. Comput. 232, 632-642 (2014).

[34] N. Meccheri, L. Fanti, "Managerial delegation contracts under centralized unionization," Manage. Decis. Econ. 35, 51-66 (2014).

[35] N.H. Miller, A.I. Pazgal, "Relative performance as a strategic commitment mechanism," Manage. Decis. Econ. 23, 51-68 (2002).

[36] C. Mira, L. Gardini, A. Barugola, J.C. Cathala, Chaotic Dynamics in Two-Dimensional Noninvertible Maps. World Scientific, Singapore (1996).

[37] T. Puu, "Chaos in duopoly pricing," Chaos Soliton. Fract. 1, 573-581 (1991).

[38] V. Salas-Fumas, "Relative performance evaluation of management: the effects on industrial competition and risk sharing," Int. J. Ind. Organ. 10, 473-489 (1992).

[39] N. Singh, X. Vives, "Price and quantity competition in a differentiated duopoly," RAND J. Econ. 15, 546-554 (1984). 
[40] S.D. Sklivas, "The strategic choice of managerial incentives," RAND J. Econ. 18, 452-458 (1987).

[41] A. van Witteloostuijn, T. Jansen, A. van Lier, "Bargaining over managerial contracts in delegation games: managerial power, contract disclosure and cartel behavior," Manage. Decis. Econ. 28, 897-904 (2007).

[42] J. Vickers, "Delegation and the theory of the firm," Econ. J. 95, 138-147 (1985). 